\title{
A Conceptual Approach towards Utilization of Technological Advancement for Coral Reef Conservation at India
}

\author{
Jebarathnam Prince Prakash Jebakumar* and Shunmugavel Ragumaran \\ National Institute of Ocean Technology, India \\ *Corresponding author: Prince Prakash jebakumar, National Institute of Ocean Technology, India
}

Submission: 眥 December 13, 2017; Published: 柴 March 26, 2018

\begin{abstract}
The unique coral reef ecosystem faces multiple stress factors from its surrounding coastal environment. Corals, an ancient marine form survived in many mass extinction events of geological past evolved to be the provider of many vital ecological services to our planet earth. Its fragility towards withstanding the pressures of fast developing coastal developments deserves conservation measures. This work conceptualizes the utility of technological advancements towards conservation of coral reef ecosystems.
\end{abstract}

\section{Introduction}

Corals are found to be the ancient marine organisms, and they evolved 540 million years ago [1]. It is sensitive to light, and temperature levels, as well as corals, survived numerous mass extinction events in geological past [2-4]. Coral reefs are home for $25 \%$ of marine species and occupy less than $1 \%$ of the world's oceans, form a unique biodiversity reserve $[5,6]$. This exceptional biodiversity has Net Primary Productivity (NPP) of $2500 \mathrm{~g} \mathrm{C} / \mathrm{m}^{2} /$ year [7] and higher than tropical rainforest of $2200 \mathrm{~g} \mathrm{C} / \mathrm{m}^{2} /$ year [6] deserves conservation efforts. Its service to our planet earth has been classified in to four major heads regulating, provisional, cultural and supporting services. The regulating services includes carbon sequestration, ocean water filtration followed by provisioning of fish/shell fish protein for humans, jobs through fishing, building materials, medical and genetic research products. The cultural services includes Tourism, recreation, aesthetics to coast and supporting services like coastal erosion prevention and serves as nursery for fish juvenile continually maintaining fish stock. It is predicted that more than $75 \%$ worldwide coral reefs were under threat hence; a conceptual approach towards adopting modern technology for coral reef conservation has been studied.

\section{Coral Reef Health Status}

According to WWF Living Planet Index [LPI,2016], a long-term measure of marine ecosystem health has declined by $36 \%$ between 1970 and 2012 with an average annual decline of 1 per cent (Figure 1). The health conditions were derived from summaries of 12,634 surveys conducted from 1975-2006 [8,9]. It suggests that coral reefs, sea-grass beds, and mangrove forests are slowly degraded by the wide range of threats facing them. It might be caused by cumulative local and global pressures [10] various local and global pressures were consolidated and tabulated hereunder (Table 1). The Indian scenario of coral reef research as well as threats to coral reefs was summarized [11,12] and tabulated hereunder (Table 2). It is prominent that population pressure and associated effects, as well as siltation, contribute major threat to Indian coral reefs. Since coastal population pressure needs administrative controls through good management practices, sedimentation required technological interventions.

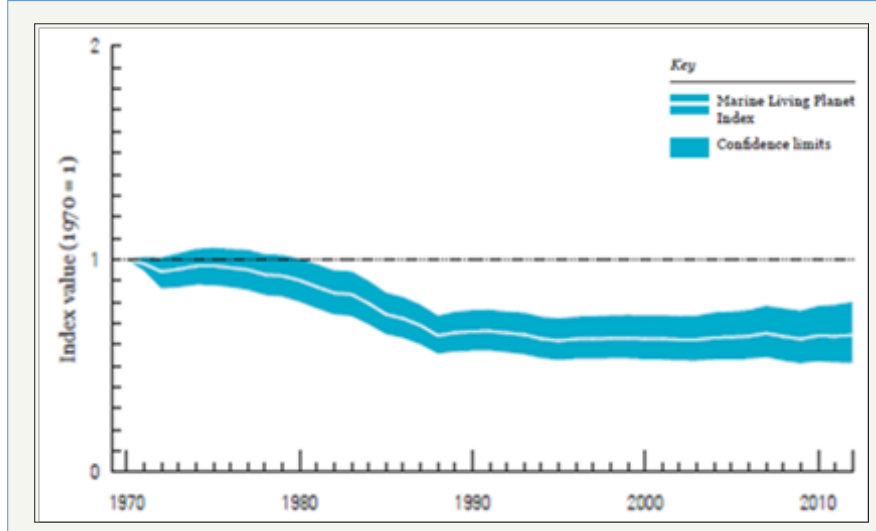

Figure 1: Coral Health Index.

\section{Impact of Sedimentation on Coral Reefs}

The issues of turbidity and sedimentation at coral reefs have received increasing attention over the past decade [13-15]. Coral reefs are recognized as structures built by animal and plant forms 
creates unique environment biologically. Their problem of siltation or sedimentation also found to be peculiar and not like most of the benthic ecosystem [16]. In general, sedimentation should be of exogenous origin from river runoff, wind or icebergs, or sediments from biological derivation such as the skeletons of plankton which settled from surface waters to the sea floor. On reef environment, the endogenous origin of sediment through death and decay of reef organism and physical, chemical and biological erosion of preexisting reef rock. This carbonate sediment spreads throughout the reef eco system and excess transported to the deep sea. However, the unprecedented coastal development along tropical shorelines is further augments the sediment load to the coral ecosystem. The exogenous sedimentation creates excess turbidity and reduces light intensity which is vital for photosynthesis and coral growth. By considering the sensitivity of corals to turbidity, the effects of dredging has been prioritized. The deleterious effects of dredging in the lagoon and reefs of Lakshadweep were studied by [17] and some specific islands [18] along Indian coastal areas. However, the ever growing economic and societal demands necessitate activities like coastal constructions, land reclamation, beach nourishment and port construction along worldwide which are depending on dredging. The impact of dredging and other causes of sediment disturbances along corals reefs are primarily related to the concentration, duration, and frequency of exposure to elevated levels of turbidity and sedimentation [19]. In general, sedimentation leads to smothering of the reef, scouring of the reef by sediment laden waters, loss of bottom area suitable for settlement of larvae and reduction of light intensity due to turbidity. The elite responses of coral reef to sedimentations were documented [19] and tabulated (Table 3).

Table 1: Different threats to coral reef ecosystem.

\begin{tabular}{|c|c|c|}
\hline S. Nos. & Local Pressures & Global Pressures \\
\hline 1 & $\begin{array}{c}\text { Overfishing, Destructive fishing (trawling, dynamite and } \\
\text { cyanide fishing) }\end{array}$ & Alien species invasion, linked to changing Ocean temperatures \\
\hline 2 & Tourism/diving damage & $\begin{array}{c}\text { Coral bleaching due to rising global sea temperatures and stronger EI } \\
\text { Nino events }\end{array}$ \\
\hline 3 & $\begin{array}{c}\text { Siltation from onshore sediment flows, often linked to } \\
\text { deforestation and farming erosion }\end{array}$ & $\begin{array}{c}\text { Ocean acidification, due to increased ocean carbon dioxide levels, } \\
\text { Weakening coral skeleton }\end{array}$ \\
\hline 4 & $\begin{array}{c}\text { Eutrophication from urban, industrial and farm pollution, } \\
\text { Coral mining for building materials }\end{array}$ & Rising sea levels 'drowning' coral in deeper waters \\
\hline
\end{tabular}

Table 2: Different threats to Indian coral reef ecosystems.

\begin{tabular}{|c|c|c|c|}
\hline Coral Reefs & Bio-Physical & Research & Perceived Threats \\
\hline Palk Bay & $\begin{array}{l}\text { Slow recovery from 60's coral } \\
\text { mining }\end{array}$ & Mainly on Bio-physical aspects & Population pressure and associated effects \\
\hline Gulf of Mannar & $\begin{array}{l}\text { Slow recovery from 60's coral } \\
\text { mining }\end{array}$ & $\begin{array}{l}\text { Bio-physical aspects; associated fauna } \\
\text { and Human activities damaging the reefs. }\end{array}$ & Population pressure and associated effects \\
\hline Andaman \& Nicobar & $\begin{array}{l}\text { Fair Excellent, Problems around } \\
\text { south island }\end{array}$ & $\begin{array}{l}\text { Bio-physical aspects; associated fauna } \\
\text { and Human activities damaging the reefs. }\end{array}$ & Siltation due to logging. Sand mining \\
\hline Lakshadweep & $\begin{array}{l}\text { Excellent off uninhabited islands } \\
\text { and endangered along habited } \\
\text { islands. }\end{array}$ & $\begin{array}{l}\text { Bio-physical aspects; associated fauna } \\
\text { and Human activities damaging the reefs. }\end{array}$ & Population pressure and associated effects \\
\hline Gulf of Kutch & $30 \%$ of the reefs are living & $\begin{array}{l}\text { Bio-physical aspects; associated fauna } \\
\text { and Human activities damaging the reefs. }\end{array}$ & $\begin{array}{l}\text { Sedimentation and siltation due to cutting } \\
\text { of mangrove forests, sand mining for } \\
\text { industrial use. Population pressure }\end{array}$ \\
\hline West coast & Unknown & Limited & Unknown \\
\hline
\end{tabular}

Table 3: Qualitative coral response to various environmental threats.

\begin{tabular}{|c|c|c|}
\hline Coral Response & Sedimentation & Turbidity \\
\hline \multicolumn{3}{|c|}{ Stress } \\
\hline Photophysiological changes & $\begin{array}{l}\text { Reduced photosynthatic efficiency zooxanthellae and auto- } \\
\text { trophic nutrition to coral }\end{array}$ & $\begin{array}{c}\text { Reduced photosynthetic efficiency zooxanthellae } \\
\text { and autotrophic nutrition to coral; switch to } \\
\text { heterotrophic feeding, ingestion of sediment } \\
\text { particles }\end{array}$ \\
\hline Changes in polypactivity & $\begin{array}{l}\text { Extrusion of mesenterial filaments following severe stress } \\
\text { Increased ciliary or polyp activity and tissue expansion in } \\
\text { some species, to remove sediment }\end{array}$ & Increased ciliary or polyp activity to feed \\
\hline Mucus production & $\begin{array}{l}\text { Increased mucus production or sheeting to remove } \\
\text { sediment }\end{array}$ & Evidence of mucus production \\
\hline \multicolumn{3}{|c|}{ Severe stress } \\
\hline
\end{tabular}




\begin{tabular}{|c|c|c|}
\hline sediment accumulation & $\begin{array}{l}\text { Accumulation of sediment on tissue of susceptible growth } \\
\text { forms due to failure of mechanisms of rejection }\end{array}$ & \\
\hline \multirow{2}{*}{ Change in coral colour } & $\begin{array}{l}\text { Change in coral colour arising from changes in the density } \\
\text { of zooxanthellae and photosynthetic pigments }\end{array}$ & $\begin{array}{l}\text { Change in coral colour arising from changes in } \\
\text { the density of zooxanthellae and photosynthetic } \\
\text { pigments }\end{array}$ \\
\hline & Paling of coral due to partial bleaching & $\begin{array}{l}\text { Darkening of coral in response to reduced light } \\
\text { due to photoacclimation }\end{array}$ \\
\hline Bleaching & \multirow{2}{*}{$\begin{array}{l}\text { Considerable whitening of corals due to the expulsion of a } \\
\text { large proportion of zooxanthellae from the colony } \\
\text { Injury to coral tissue, loss of polyps and partial mortality of } \\
\text { the colony } \\
\text { Decrease in (live) coral cover }\end{array}$} & \\
\hline Partial mortality & & $\begin{array}{l}\text { Not Known } \\
\text { Injury to coral tissue, loss of polyps and partial } \\
\text { mortality of the colony Decrease in (live) coral cover }\end{array}$ \\
\hline \multirow{7}{*}{ Mortality } & $\begin{array}{l}\text { Mortality of small-sized colonies and partial mortality of } \\
\text { large corals }\end{array}$ & \\
\hline & Mortality of susceptible species and size classes & \\
\hline & Decreased density, diversity and coral cover & Decreased density ,diversity and coral cover \\
\hline & Changes in community structure & Changes in community structure \\
\hline & Wide-spread mortality of corals & Wide-spread mortality of corals \\
\hline & Major decreases in density, diversity and coral cover & $\begin{array}{c}\begin{array}{c}\text { Major decreases in density, diversity and coral } \\
\text { cover }\end{array}\end{array}$ \\
\hline & $\begin{array}{c}\text { Dramatic changes in community structure, and shifts } \\
\text { towards the dominance of non-coral species, such as } \\
\text { sponges and algae }\end{array}$ & $\begin{array}{l}\text { Dramatic changes in community structure, and } \\
\text { shifts towards the dominance of non-coral species } \\
\text { such as sponges and algae }\end{array}$ \\
\hline
\end{tabular}

However, the sensitivity of a coral reef to dredging impacts and its resilience depends on the ambient conditions habitually experienced. To understand the background concentrations of sedimentation among undisturbed reef environments were studied by $[20,21]$ it mean value ranges from less than 1 to about $110 \mathrm{mg}$ $\mathrm{cm}^{-2} \mathrm{~d}^{-1}$ or $<10 \mathrm{mg} \mathrm{L}^{-1}$ of suspended sediment concentration. In India, sedimentation rate studies are limited, and the pristine reefs

Table 4: Quantitative coral response to turbidity. of Lakshadweep islands recorded 2.69 to $124.49 \mathrm{mgcm}^{-2} \mathrm{~d}^{-1}$ [21]. The highest value was related to monsoonal effects of terrigenous runoff [15]. The data from Palk Bay during May to October 2004 varied between 1 to $42 \mathrm{mgcm}^{-2} \mathrm{~d}^{-1}$ [18]. The highest value was recorded during June 2004 heavy precipitation period. However extreme event like a tsunami of 26 December 2004 has elevated the sedimentation rate to $54 \mathrm{mgcm}^{-2} \mathrm{~d}^{-1}$ in the Palkbay area [22].

\begin{tabular}{|c|c|c|c|c|c|}
\hline Coral Species & $\begin{array}{l}\text { Turbidity Level } \\
\text { (Tested) }\end{array}$ & Response & Growth Form & $\begin{array}{l}\text { Calyx } \\
(\mathrm{Mm})\end{array}$ & References \\
\hline $\begin{array}{l}\text { Acropora cervicornis } \\
\text { (Lamarck,1816) }\end{array}$ & $\begin{array}{l}\text { Severe light reduction } \\
\text { (shading) for } 5 \text { weeks }\end{array}$ & $\begin{array}{c}\text { Mass bleaching (3 weeks), } \\
\text { mortality/algal cover ( } \\
\text { weeks), no recovery ( } 8 \\
\text { months) }\end{array}$ & B & 1 & Rogers [23] \\
\hline $\begin{array}{l}\text { Acropora cervicornis } \\
\text { (Lamarck,1816) }\end{array}$ & $50 \mathrm{mg} / \mathrm{l}(96 \mathrm{~h})$ & No effect & B & 1 & Thompson [24] \\
\hline $\begin{array}{l}\text { Acropora cervicornis } \\
\text { (Lamarck,1816) }\end{array}$ & $150 \mathrm{mg} / \mathrm{l}(96 \mathrm{~h})$ & $\begin{array}{l}\text { Polyp retraction, mucus } \\
\text { production but no mortality }\end{array}$ & B & 1 & Thompson [24] \\
\hline $\begin{array}{l}\text { Acropora cervicornis } \\
\text { (Lamarck,1816) }\end{array}$ & 476rng/l (96h) & Partial mortality after $96 \mathrm{~h}$. & B & 1 & Thompson [24] \\
\hline $\begin{array}{l}\text { Acropora cervicornis } \\
\text { (Lamarck,1816) }\end{array}$ & $\begin{array}{l}\text { Total shading ( } 3 \\
\text { weeks) }\end{array}$ & $\begin{array}{l}\text { Bleaching and mortality, no } \\
\text { recovery }\end{array}$ & B & 1 & Nieuwaal [25] \\
\hline $\begin{array}{l}\text { Acropora cervicornis } \\
\text { (Lamarck,1816) }\end{array}$ & $\begin{array}{c}25 \mathrm{mg} / \mathrm{I} \text { (drilling mud) } \\
(24 \mathrm{~h})\end{array}$ & $\begin{array}{l}62 \% \text { Decrease in calcification } \\
\text { rate }\end{array}$ & B & 1 & Kendall et al. [26] \\
\hline $\begin{array}{l}\text { Acropora cervicornis } \\
\text { (Lamarck,1816) }\end{array}$ & $\begin{array}{l}100 \mathrm{mg} / \mathrm{l} \text { (drilling } \\
\mathrm{mud})(24 \mathrm{~h})\end{array}$ & $\begin{array}{l}50 \% \text { Decline in soluble tissue } \\
\text { protein }\end{array}$ & B & 1 & Kendall et al. [26] \\
\hline $\begin{array}{l}\text { Acropora cervicornis } \\
\text { (Lamarck,1816) }\end{array}$ & $\begin{array}{l}50 \text { and } 100 \mathrm{mg} / \mathrm{I} \\
\text { (kaolin,24h) }\end{array}$ & $\begin{array}{l}\text { Reduced calcification rate and } \\
\text { free amino acids at } 100 \mathrm{mg} / \mathrm{I} \\
\text { (recovery in } 48 \mathrm{~h} \text { ) }\end{array}$ & B & 1 & Kendall et al. [27] \\
\hline $\begin{array}{l}\text { Acropora cervicornis } \\
\text { (Lamarck,1816) }\end{array}$ & $1000 \mathrm{mg} / \mathrm{I}$ (for $65 \mathrm{~h}$ ) & Mortality of colonies & B & 1 & $\begin{array}{c}\text { Thompson \& Bright } \\
{[28]}\end{array}$ \\
\hline Acropora digitifera (larvae) & $\begin{array}{l}50-100 \mathrm{mg} / \mathrm{l}(\mathrm{lab} \text { and } \\
\text { feld tests) }\end{array}$ & $\begin{array}{c}\text { Adverse effects on } \\
\text { fertilisation, larval survival } \\
\text { and settlement }\end{array}$ & - & 1 & Gilmour [29] \\
\hline
\end{tabular}




\begin{tabular}{|c|c|c|c|c|c|}
\hline Coral Species & $\begin{array}{l}\text { Turbidity Level } \\
\text { (Tested) }\end{array}$ & Response & Growth Form & $\begin{array}{l}\text { Calyx } \\
\text { (Mm) }\end{array}$ & References \\
\hline $\begin{array}{l}\text { Acropora millepora } \\
\text { (Ehrenberg,1834) }\end{array}$ & 1-30mg/I SPM (hours) & $\begin{array}{l}\text { Increased feeding capacity at } \\
\text { high SPM concentrations }\end{array}$ & B & 1 & Anthony [30] \\
\hline $\begin{array}{l}\text { Acropora millepora } \\
\text { (Ehrenberg,1834) }\end{array}$ & 1-30mg/l SPM (days) & $\begin{array}{l}\text { Increasing contribution of } \\
\text { heterotrophy at high SPM } \\
\text { concentration }\end{array}$ & B & 1 & Anthony [31] \\
\hline $\begin{array}{l}\text { Acropora millepora } \\
\text { (Ehrenberg,1834) }\end{array}$ & $\begin{array}{c}1,3,10,30 \text { and to } \\
\text { Omg/l TSS (16 weeks) }\end{array}$ & $\begin{array}{l}\text { Full colony mortality at to0 } \\
\text { mg/I for } 12 \text { weeks ( } 50 \% \\
\text { mortality after } 4 \text { weeks) }\end{array}$ & B & 1 & $\begin{array}{l}\text { Negri et al. [32] \& } \\
\text { Flores et al. [33] }\end{array}$ \\
\hline Acropora nobilis (Dana, 1846) & 10mg/I (42 days) & $\begin{array}{l}\text { Increased survival from } \\
\text { high temperature treatment } \\
\text { compared to control }\end{array}$ & $\mathrm{L}$ & 1.5 & Anthony et al. [34] \\
\hline Acropora spp. & $\begin{array}{l}170 \mathrm{mg} / \mathrm{I} \text { (hours) of } \\
\text { marine snow/SPM }\end{array}$ & $\begin{array}{l}\text { Mucus production in response } \\
\text { to flocculation }\end{array}$ & - & - & $\begin{array}{c}\text { Fabricius \& Wolanski } \\
{[35]}\end{array}$ \\
\hline $\begin{array}{l}\text { Agaricia agaricites } \\
\text { (Linnaeus,1758) }\end{array}$ & $\begin{array}{l}\text { Severe light reduction } \\
\text { (shading) for } 5 \text { weeks }\end{array}$ & $\begin{array}{c}\text { Partial bleaching after } 5 \\
\text { weeks, recovery within weeks }\end{array}$ & $\mathrm{L}$ & 5 & Rogers [23] \\
\hline $\begin{array}{c}\text { Agaricia agaricites } \\
\text { (Linnaeus,1758) }\end{array}$ & $50 \mathrm{mg} / \mathrm{l}(96 \mathrm{~h})$ & No effect & $\mathrm{L}$ & 5 & Thompson [24] \\
\hline $\begin{array}{l}\text { Agaricia agaricites } \\
\text { (Linnaeus,1758) }\end{array}$ & $150 \mathrm{mg} / \mathrm{I}(96 \mathrm{~h})$ & $\begin{array}{l}\text { Polyp retraction, mucus } \\
\text { production but no mortality }\end{array}$ & $\mathrm{L}$ & 5 & Thompson [24] \\
\hline $\begin{array}{c}\text { Agaricia agaricites } \\
\text { (Linnaeus,1758) }\end{array}$ & $476 \mathrm{mg} / \mathrm{I}(96 \mathrm{~h})$ & Mortality after $65 \mathrm{~h}$ & $\mathrm{~L}$ & 5 & Thompson [24] \\
\hline $\begin{array}{l}\text { Agaricia agaricites } \\
\text { (Linnaeus,1758) }\end{array}$ & $<1 \%$ SI (several days) & $\begin{array}{l}33 \% \text { Decrease in calcification } \\
\text { rate (for }>1 \text { month),but } \\
\text { survival }\end{array}$ & $\mathrm{L}$ & 5 & Bak [36] \\
\hline $\begin{array}{c}\text { Agaricia agaricites } \\
\text { (Linnaeus,1758) }\end{array}$ & $1000 \mathrm{mg} / \mathrm{I}$ (for $65 \mathrm{~h}$ ) & Mortality of colonies & $\mathrm{L}$ & 5 & $\begin{array}{c}\text { Thompson \& Bright } \\
{[28]}\end{array}$ \\
\hline $\begin{array}{l}\text { Cladocora arbuscula } \\
\text { (Lesueur,1812) }\end{array}$ & $\begin{array}{c}\text { 49, tol, } 165 \text { and } \\
199 \mathrm{mg} / \mathrm{I}(10-20 \text { days })\end{array}$ & $\begin{array}{l}\text { No effect on growth rate } \\
\text { or survival (to d), minor } \\
\text { bleaching }(20 \mathrm{~d})\end{array}$ & B & 4 & Rice \& Hunter [37] \\
\hline $\begin{array}{l}\text { Colpophy Uianatans } \\
\text { (Houttuyn,1772) }\end{array}$ & $\begin{array}{l}\text { Severe light reduction } \\
\text { (shading) for } 5 \text { weeks }\end{array}$ & $\begin{array}{l}\text { Partial bleaching ( } 5 \text { weeks), } \\
\text { limited recovery \& some algal } \\
\text { growth (15 weeks) }\end{array}$ & M & 25 & Rogers [23] \\
\hline $\begin{array}{l}\text { Dichacaenia stokes (Milne } \\
\text { Edward \& Haime, 1848) }\end{array}$ & $\begin{array}{c}\text { 0-2 NTU and 7-9 NTU } \\
\text { (weeks) }\end{array}$ & No effect on P:R ratio & M & 11 & $\begin{array}{c}\text { Telesnicki \& Goldberg } \\
{[38]}\end{array}$ \\
\hline $\begin{array}{l}\text { Dichacaenia stokes (Milne } \\
\text { Edward \& Haime, 1848) }\end{array}$ & 14-16 NTU (weeks) & $\begin{array}{l}\text { Mucus production, } P: R \text { ratio } \\
<1 \text { after } 6 \text { days exposure }\end{array}$ & M & 11 & $\begin{array}{c}\text { Telesnicki \& Goldberg } \\
{[38]}\end{array}$ \\
\hline $\begin{array}{l}\text { Dichacaenia stokes (Milne } \\
\text { Edward \& Haime, 1848) }\end{array}$ & 28-30 NTU (weeks) & $\begin{array}{l}\text { Mucus production, } \mathrm{P}: \mathrm{R} \text { ratio } \\
<1 \text { after } 3 \text { days exposure }\end{array}$ & M & 11 & $\begin{array}{c}\text { Telesnicki \& Goldberg } \\
{[38]}\end{array}$ \\
\hline $\begin{array}{l}\text { Dichacaenia stokes (Milne } \\
\text { Edward \& Haime, 1848) }\end{array}$ & $50-150-476 \mathrm{mg} / \mathrm{l}(96 \mathrm{~h})$ & $\begin{array}{l}\text { No effect at } 50 \text { and } 150 \mathrm{mg} / \mathrm{l}: \\
\text { extreme sublethal stress but } \\
\text { survival at } 476 \mathrm{mg} / \mathrm{l}\end{array}$ & M & 11 & Thompson [24] \\
\hline $\begin{array}{l}\text { Dichacaenia stokes (Milne } \\
\text { Edward \& Haime, 1848) }\end{array}$ & $1000 \mathrm{mg} / \mathrm{l}$ (for $65 \mathrm{~h}$ ) & No mortality & M & 11 & $\begin{array}{l}\text { Thompson \& Bright } \\
{[28]}\end{array}$ \\
\hline $\begin{array}{l}\text { Diploria labyrinthiformis } \\
\text { (Linnaeus, 1758) }\end{array}$ & $\begin{array}{l}\text { Severe light reduction } \\
\text { (shading) for } 5 \text { weeks }\end{array}$ & $\begin{array}{c}\text { Substantial bleaching ( } 5 \\
\text { weeks), no recovery \& soalgal } \\
\text { growth (15 weeks) }\end{array}$ & M & 8 & Rogers [23] \\
\hline Eusmilia fastigiata (Pallas, 1766) & $\begin{array}{l}\text { severe light reduction } \\
\text { (shading) for } 5 \text { weeks }\end{array}$ & No visible effects & M & 12 & Rogers [23] \\
\hline Favia favus (Forskal, 1775) & $\begin{array}{l}\text { Light reduced to } 50 \% \\
\text { and } 25 \% \text { PAR (surface) }\end{array}$ & $\begin{array}{c}\text { Severely diminished } \\
\text { productivity, increased carbon } \\
\text { loss and mucus }\end{array}$ & M & 14 & Riegl \& Branch [39] \\
\hline Favites pentagona (Esper, 1794) & $\begin{array}{l}\text { Light reduced to } 50 \% \\
\text { and } 25 \% \text { PAR (surface) }\end{array}$ & $\begin{array}{c}\text { Severely diminished } \\
\text { productivity, increased carbon } \\
\text { loss and mucus }\end{array}$ & M & 7 & Riegl \& Branch [39] \\
\hline Fungiidae (mushroom corals) & & $\begin{array}{l}\text { Adapted to highly turbid } \\
\text { environments }\end{array}$ & & & $\begin{array}{c}\text { Dikou \& Van Woesik } \\
{[40,41]}\end{array}$ \\
\hline
\end{tabular}




\begin{tabular}{|c|c|c|c|c|c|}
\hline Coral Species & $\begin{array}{l}\text { Turbidity Level } \\
\text { (Tested) }\end{array}$ & Response & Growth Form & $\begin{array}{l}\text { Calyx } \\
\text { (Mm) }\end{array}$ & References \\
\hline $\begin{array}{l}\text { Galaxea fascicularis } \\
\text { (Linnaeus,1767) }\end{array}$ & $\begin{array}{l}>40 \mathrm{NTU} \text { (cAD d), at } \\
\text { times up to } 175 \mathrm{NTU}\end{array}$ & $\begin{array}{l}\text { Shift from autotrophy to } \\
\text { heterotrophy (reversible) }\end{array}$ & $\mathrm{C}$ & 8 & Larcombe et al. [42] \\
\hline $\begin{array}{l}\text { Goniastrea retiformis } \\
\text { (Lamarck,1816) }\end{array}$ & $\begin{array}{l}\text { Shading (equivalent to } \\
16 \mathrm{mg} / \mathrm{l}^{-2} \text { ) months }\end{array}$ & $\begin{array}{l}\text { Increased particle feeding } \\
\text { \&heterotrophy;survival and } \\
\text { tissue gains }\end{array}$ & M & 4 & $\begin{array}{c}\text { Anthony \& Fabricius } \\
{[43]}\end{array}$ \\
\hline $\begin{array}{l}\text { Goniastrea retiformis } \\
\text { (Lamarck.1816) }\end{array}$ & $\begin{array}{l}\text { 1-30mg/1 SPM } \\
\text { (weeks) }\end{array}$ & $\begin{array}{l}\text { Gained tissue \& skeletal mass } \\
\text { (all treatments); increasing } \\
\text { heterotrophy }\end{array}$ & M & 4 & $\begin{array}{c}\text { Anthony \& Fabricius } \\
{[43]}\end{array}$ \\
\hline $\begin{array}{l}\text { Goniastrea retiformis } \\
\text { (Lamarck.1816) }\end{array}$ & $\begin{array}{l}\text { 1-16mg/I suspended } \\
\text { matter ( } 8 \text { weeks) }\end{array}$ & $\begin{array}{c}\text { Increased growth rate as } \\
\text { function of SPM concentration }\end{array}$ & M & 4 & Anthony [44] \\
\hline $\begin{array}{l}\text { Goniastrea retiformis } \\
\text { (Lamarck.1816) }\end{array}$ & $\begin{array}{l}\text { Shading (equiv. } \\
16 \mathrm{mg} / \mathrm{I} \text { at } 4 \mathrm{~m})(8 \\
\text { weeks }\end{array}$ & $\begin{array}{l}\text { Significant reduction in } \\
\text { growth rate }\end{array}$ & M & 4 & Anthony [44] \\
\hline $\begin{array}{l}\text { Gorgonia flabellum (Linnaeus, } \\
1758 \text { ) }\end{array}$ & $\begin{array}{l}\text { Severe light reduction } \\
\text { (shading) for } 5 \text { weeks }\end{array}$ & No visible effects & So & & Rogers [23] \\
\hline Gorgonians \& soft corals & - & Very tolerant to high turbidity & - & - & $\begin{array}{c}\text { Fabricius \& Dommisse } \\
{[45]}\end{array}$ \\
\hline $\begin{array}{l}\text { Gyrosmilia interrupta } \\
\text { (Ehrenberg. 1834) }\end{array}$ & $\begin{array}{l}\text { Light reduced to } 50 \% \\
\text { and } 25 \% \text { PAR (surface) }\end{array}$ & $\begin{array}{l}\text { Severely diminished } \\
\text { productivity. Increased } \\
\text { carbon loss and mucus }\end{array}$ & $\mathrm{M} / \mathrm{E}$ & 16 & Riegl \& Branch [39] \\
\hline $\begin{array}{l}\text { Isophyllia sinuosa (Ellis } \\
\text { \&Selander, 1786) }\end{array}$ & $\begin{array}{c}\text { 49.101.165 and } \\
199 \mathrm{mg} / \mathrm{l}(10-20 \text { days })\end{array}$ & $\begin{array}{l}\text { No effect on growth rate or } \\
\text { survival after } 10 \mathrm{~d} \text {. minor } \\
\text { bleaching after } 20 \mathrm{~d}\end{array}$ & $\mathrm{~N}$ & 15 & Rice \& Hunter [37] \\
\hline Leptastrea sp. & - & Well adapted to turbid waters & - & - & $\begin{array}{c}\text { Dikou \& Van Woesik } \\
{[40,41]}\end{array}$ \\
\hline $\begin{array}{l}\text { Lophytum depress (Tixier } \\
\text { Durivault, 1957) }\end{array}$ & $\begin{array}{l}\text { Light reduced to } 50 \% \\
\text { and } 25 \% \text { PAR (surface) }\end{array}$ & $\begin{array}{l}\text { Severely diminished } \\
\text { productivity, increased } \\
\text { carbon loss and mucus }\end{array}$ & So & - & Riegl \& Branch [39] \\
\hline $\begin{array}{l}\text { Lophytum depress (Tixier } \\
\text { Durivault, 1957) }\end{array}$ & $\begin{array}{l}\text { Light reduced to } 50 \% \\
\text { and } 25 \% \text { PAR (surface) }\end{array}$ & $\begin{array}{l}\text { Severely diminished } \\
\text { productivity, increased } \\
\text { carbon loss and mucus }\end{array}$ & So & - & Riegl \& Branch [39] \\
\hline $\begin{array}{l}\text { Madracis auretenra (Locke, Well } \\
\quad \& \text { Coates, 2007) }\end{array}$ & <1\% SI (several days) & $\begin{array}{l}33 \% \text { Decrease in calcification } \\
\text { rate (for }>1 \text { month),but } \\
\text { survival }\end{array}$ & B & 1 & Bak [36] \\
\hline $\begin{array}{l}\text { Manicina Areolata (Linneaus, } \\
\text { 1758) }\end{array}$ & $\begin{array}{c}\text { 49.101,165 and } \\
199 \mathrm{mg} / \mathrm{I}(10-20 \text { days })\end{array}$ & $\begin{array}{l}\text { No effect on growth rate or } \\
\text { survival after } 10 \mathrm{~d} \text {. minor } \\
\text { bleaching after } 20 \mathrm{~d}\end{array}$ & M & 14 & Rice \& Hunter [37] \\
\hline $\begin{array}{l}\text { Meandrina meandrites } \\
\text { (Linnaeus, 1758) }\end{array}$ & $\begin{array}{l}\text { 0-2 NTU and 7-9 NTU } \\
\text { (weeks) }\end{array}$ & No effect on $\mathrm{P}: \mathrm{R}$ ratio & $\mathrm{M} / \mathrm{E}$ & 15 & $\begin{array}{c}\text { Telesnicki \& Goldberg } \\
{[38]}\end{array}$ \\
\hline $\begin{array}{l}\text { Meandrina meandrites } \\
\text { (Linnaeus, 1758) }\end{array}$ & 14-16 NTU (weeks) & $\begin{array}{c}\text { Mucus production, } \mathrm{P}: \mathrm{R} \text { ratio }< \\
1 \text { after } 6 \text { days exposure }\end{array}$ & $\mathrm{M} / \mathrm{E}$ & 15 & $\begin{array}{c}\text { Telesnicki \& Goldberg } \\
{[38]}\end{array}$ \\
\hline $\begin{array}{l}\text { Meandrina meandrites } \\
\text { (Linnaeus, 1758) }\end{array}$ & 28-30 NTU (weeks) & $\begin{array}{c}\text { Mucus production, } \mathrm{P}: \mathrm{R} \text { ratio }< \\
1 \text { after } 3 \text { days exposure }\end{array}$ & $\mathrm{M} / \mathrm{E}$ & 15 & $\begin{array}{c}\text { Telesnicki \& Goldberg } \\
{[38]}\end{array}$ \\
\hline $\begin{array}{l}\text { Millepora alcicornis } \\
\text { (Linnaeus,1758) }\end{array}$ & $\begin{array}{l}\text { Severe light reduction } \\
\text { (shading) for } 5 \text { weeks }\end{array}$ & $\begin{array}{l}\text { Partial bleaching ( } 5 \text { weeks). } \\
\text { algal growth ( } 6 \text { weeks). no } \\
\text { recovery of damaged tissue }\end{array}$ & B & 0.5 & Rogers [23] \\
\hline $\begin{array}{l}\text { Montastraea annularis (Ellis } \\
\text { \&Selander, 1786) }\end{array}$ & $\begin{array}{l}\text { Severe light reduction } \\
\text { (shading) for } 5 \text { weeks }\end{array}$ & $\begin{array}{c}\text { Substantial bleaching (5 } \\
\text { weeks). partial recovery (6-8 } \\
\text { weeks). some algae/mucus }\end{array}$ & $\mathrm{M} / \mathrm{E}$ & 5 & Rogers [23] \\
\hline $\begin{array}{l}\text { Montastraea annularis (Ellis } \\
\text { \&Selander, 1786) }\end{array}$ & $50 \mathrm{~m} / \mathrm{l}(96 \mathrm{~h})$ & No effect & $\mathrm{M} / \mathrm{E}$ & 5 & Thompson [24] \\
\hline $\begin{array}{l}\text { Montastraea annularis (Ellis } \\
\text { \&Selander, 1786) }\end{array}$ & $150 \mathrm{mg} / \mathrm{I}(96 \mathrm{~h})$ & $\begin{array}{l}\text { Polyp retraction. mucus } \\
\text { production but no mortality }\end{array}$ & $\mathrm{M} / \mathrm{E}$ & 5 & Thompson [24] \\
\hline $\begin{array}{l}\text { Montastraea annularis (Ellis } \\
\text { \&Selander, 1786) }\end{array}$ & $476 \mathrm{mg} / \mathrm{l}(96 \mathrm{~h})$ & Mortality after $65 \mathrm{~h}$ & $M / E$ & 5 & Thompson [24] \\
\hline $\begin{array}{l}\text { Montastraea annularis (Ellis } \\
\quad \text { \&Selander, 1786) }\end{array}$ & 100mg/I (6-weeks) & $\begin{array}{l}\text { Major sublethal effects } \\
\text { (photosynthesis, respiration. } \\
\text { calcification \&nutr.uptake) }\end{array}$ & $\mathrm{M} / \mathrm{E}$ & 5 & $\begin{array}{l}\text { Szmant-Froelich et al. } \\
{[46]}\end{array}$ \\
\hline
\end{tabular}




\begin{tabular}{|c|c|c|c|c|c|}
\hline Coral Species & $\begin{array}{l}\text { Turbidity Level } \\
\text { (Tested) }\end{array}$ & Response & Growth Form & $\begin{array}{l}\text { Calyx } \\
(\mathrm{Mm})\end{array}$ & References \\
\hline $\begin{array}{c}\text { Montastraea annularis (Ellis } \\
\text { \&Selander, 1786) }\end{array}$ & $1-10 \mathrm{mg} / 1$ (6 weeks) & $\begin{array}{l}\text { Only (some) effect on feeding } \\
\text { response }\end{array}$ & $\mathrm{M} / \mathrm{E}$ & 5 & $\begin{array}{l}\text { Szmant-Froelich et al. } \\
{[46]}\end{array}$ \\
\hline $\begin{array}{l}\text { Montastraea annularis (Ellis } \\
\quad \text { \&Selander, 1786) }\end{array}$ & $525 \mathrm{mg} / \mathrm{I}$ & $\begin{array}{l}\text { Decreased net production } \\
\& \text { tissue ChI, increased } \\
\text { respiration \& mucus }\end{array}$ & $\mathrm{M} / \mathrm{E}$ & 5 & Dallmeyer et al. [47] \\
\hline $\begin{array}{l}\text { Montastraea annularis (Ellis } \\
\text { \&Selander, 1786) }\end{array}$ & $1000 \mathrm{mg} / \mathrm{l}$ (for $65 \mathrm{~h}$ ) & Mortality of colonies & $\mathrm{M} / \mathrm{E}$ & 5 & $\begin{array}{c}\text { Thompson \& Bright } \\
{[28]}\end{array}$ \\
\hline $\begin{array}{l}\text { Montastraea cavernosa } \\
\text { (Linnaeus, 1767) }\end{array}$ & $\begin{array}{l}\text { Severe light reduction } \\
\text { (shading) for } 5 \text { weeks }\end{array}$ & No visible effects & M & 11 & Rogers [23] \\
\hline $\begin{array}{l}\text { Montipora aequituberculata } \\
\text { (Bernard, 1897) }\end{array}$ & - & $\begin{array}{l}\text { Common on shallow. turbid } \\
\text { inshore fringing reefs }\end{array}$ & $\mathrm{F}$ & 0.6 & Stafford-Smith [48] \\
\hline $\begin{array}{l}\text { Montipora aequituberculata } \\
\text { (Bernard, 1897) }\end{array}$ & $\begin{array}{l}\text { 1. } 3,10,30 \text { and } 100 \\
\text { mg/I TSS (16 weeks) }\end{array}$ & $\begin{array}{c}\text { Full colony mortality at } 30 \\
\text { mgfl after } 12 \text { weeks ( } 50 \% \\
\text { mortality after } 4 \text { weeks) }\end{array}$ & $\mathrm{F}$ & 0.6 & $\begin{array}{l}\text { Negri et al. [32] \& } \\
\text { Flores et al. [33] }\end{array}$ \\
\hline Montipora capitate (Dana, 1846) & $\begin{array}{l}\text { Light reduction from } \\
57 \text { to } 44 \% \text { SI (field; } \\
\text { hours) }\end{array}$ & $\begin{array}{l}\text { Photophysiologicalsublethal } \\
\text { response; } 1.4 \text { times lower } \\
\text { rETR. higher Fv/Fm }\end{array}$ & B & 1 & Piniak \& Storlazzi [49] \\
\hline Montipora digitata (Dana. 1846) & 1-30mgjl SPM (hours) & $\begin{array}{l}\text { Increased feeding capacity at } \\
\text { high SPM concentrations }\end{array}$ & B & 1 & Anthony [30] \\
\hline Montipora digitata (Dana. 1846) & $\begin{array}{l}>95 \% \text { shading } \\
\text { (transplanted } \\
\text { Hoeghinto caves) }\end{array}$ & $\begin{array}{c}\text { Survival/acclimation, reduced } \\
\text { photosynthetic rate }\end{array}$ & $\mathrm{L}$ & 1 & $\begin{array}{l}\text { Anthony \& Hoegh } \\
\text { Guldberg [50] }\end{array}$ \\
\hline Montipora digitata (Dana, 1846) & $\begin{array}{l}70 \% \text { light reduction } \\
\text { (permanent } \\
\text { transplantation) }\end{array}$ & $\begin{array}{l}\text { Complete photo acclimation } \\
\text { within } 3 \text { weeks }\end{array}$ & $\mathrm{L}$ & 1 & $\begin{array}{l}\text { Anthony \& Hoegh- } \\
\text { Guldberg [50] }\end{array}$ \\
\hline $\begin{array}{l}\text { Montipora verrucosa (Lamarck, } \\
\text { 1816) }\end{array}$ & $\begin{array}{l}8 \text { and } 20 \text { rngjl } \\
\text { (modelling) }\end{array}$ & $\begin{array}{c}\text { Reduced photosynthesis at } 8 \\
\text { mg/I; negative energy balance } \\
\text { at } 20 \mathrm{mg} / \mathrm{I}\end{array}$ & $\mathrm{M} / \mathrm{L}$ & 1 & $\mathrm{Te}[51]$ \\
\hline Montipora sp. & - & Well adapted to turbid waters & - & - & $\begin{array}{c}\text { Dikou \& Van Woesik } \\
{[40,41]}\end{array}$ \\
\hline Mussaangulosa (Pallas. 1766) & $\begin{array}{l}\text { Severe light reduction } \\
\text { (shading) for } 5 \text { weeks }\end{array}$ & $\begin{array}{l}\text { No visible effects ( } 1 \text { colony } \\
\text { showing minor bleaching } \\
\text { after } 8 \text { weeks) }\end{array}$ & M & 40 & Rogers [23] \\
\hline $\begin{array}{l}\text { Pectinialactuca (Pallas. 1766) } \\
\text { (larvae) }\end{array}$ & $\begin{array}{l}\text { 6, } 43 \text { and } 169 \mathrm{mg} / \mathrm{I} \\
\text { (lab test) }\end{array}$ & $\begin{array}{l}\text { Adverse effects on } \\
\text { fertilisation success and } \\
\text { embryo development }\end{array}$ & - & - & Erftemeijer et al. [52] \\
\hline Pectinia sp. & - & Well adapted to turbid waters & - & - & $\begin{array}{c}\text { Dikou \& Van Woesik } \\
{[40,41]}\end{array}$ \\
\hline $\begin{array}{l}\text { Phyllangia Americana (Milne } \\
\text { Edwards \&Hairne, 1849) }\end{array}$ & $\begin{array}{l}\text { 49.101.165 and } 199 \\
\mathrm{mg} / \mathrm{I}(10-20 \text { days })\end{array}$ & $\begin{array}{l}\text { No effect on growth rate or } \\
\text { survival after } 10 \mathrm{~d} \text {, minor } \\
\text { bleaching after } 20 \mathrm{~d}\end{array}$ & E & 9 & Rice \& Hunter [37] \\
\hline $\begin{array}{l}\text { Platygyra daedalea (Ellis } \\
\text { \&Solander, 1786) }\end{array}$ & $\begin{array}{l}\text { Light reduced to } 50 \% \\
\text { and } 25 \% \text { PAR (surface) }\end{array}$ & $\begin{array}{c}\text { Severely diminished } \\
\text { productivity, increased carbon } \\
\text { loss and mucus }\end{array}$ & M & 5 & Riegl \& Branch [39] \\
\hline $\begin{array}{l}\text { Pocilloporadamicomis } \\
\text { (Linnaeus, 1758) }\end{array}$ & 1-30 mg/I SPM (hours) & $\begin{array}{l}\text { Increased feeding capacity at } \\
\text { high SPM concentrations }\end{array}$ & B & 1.1 & Anthony [30] \\
\hline $\begin{array}{l}\text { Pocilloporadamicomis } \\
\text { (Linnaeus, 1758) }\end{array}$ & 1-30mg/I SPM (days) & $\begin{array}{l}\text { Increasing contribution of } \\
\text { heterotrophy at high SPM } \\
\text { concentration }\end{array}$ & B & 1.1 & Anthony [31] \\
\hline $\begin{array}{l}\text { Pocilloporadamicomis } \\
\text { (Linnaeus, 1758) (larvae) }\end{array}$ & $\begin{array}{l}\text { 10,100,1000Mg/l } \\
\text { (modelling) }\end{array}$ & $\begin{array}{l}\text { Reverse metamorphosis } \\
\text { (reduced settlement success) } \\
\text { at } 100 \text { and } 1000 \mathrm{mg} / \mathrm{I}\end{array}$ & B & 1.1 & $\mathrm{Te}[51]$ \\
\hline $\begin{array}{l}\text { Pocilloporadamicomis } \\
\text { (Linnaeus, 1758) }\end{array}$ & - & $\begin{array}{c}\text { Characteristic of turbid } \\
\text { waters }\end{array}$ & B & 1.1 & $\begin{array}{l}\text { Dikou \& Van Woesik } \\
{[40,41]}\end{array}$ \\
\hline
\end{tabular}




\begin{tabular}{|c|c|c|c|c|c|}
\hline Coral Species & $\begin{array}{l}\text { Turbidity Level } \\
\text { (Tested) }\end{array}$ & Response & Growth Form & $\begin{array}{l}\text { Calyx } \\
(\mathrm{Mm})\end{array}$ & References \\
\hline $\begin{array}{c}\text { Porites astreoides (Lamarck, } \\
\text { 1816) }\end{array}$ & $\begin{array}{c}50-150-476 \mathrm{mg} / \mathrm{I}(96 \\
\mathrm{h})\end{array}$ & $\begin{array}{l}\text { No effect at } 50 \text { and } 150 \mathrm{mg} / \mathrm{l} \text { : } \\
\text { extreme sublethal stress (but } \\
\text { survival) at } 476 \mathrm{mg} / \mathrm{I}\end{array}$ & $\mathrm{M} / \mathrm{E}$ & 1.5 & Thompson [24] \\
\hline $\begin{array}{c}\text { Porites astreoides (Lamarck, } \\
\text { 1816) }\end{array}$ & $<1 \%$ SI (several days) & Bleaching and mortality & $\mathrm{M} / \mathrm{E}$ & 1.5 & Bak [36] \\
\hline $\begin{array}{c}\text { Porites astreoides (Lamarck, } \\
\text { 1816) }\end{array}$ & $1000 \mathrm{mg} / \mathrm{I}$ (for $65 \mathrm{~h}$ ) & No mortality & $\mathrm{M} / \mathrm{E}$ & 1.5 & $\begin{array}{c}\text { Thompson \& Bright } \\
{[28]}\end{array}$ \\
\hline Porites cylindrica (Dana, 1821) & $\begin{array}{l}\text { Shading (equivalent to } \\
16 \mathrm{mg} / \mathrm{l} \text { ) - } 2 \text { months }\end{array}$ & $\begin{array}{l}\text { Energy deficiency/C-Ioss } \\
\text { not compensated by particle } \\
\text { feeding; sublethal stress }\end{array}$ & M & 1.5 & $\begin{array}{c}\text { Anthony \& Fabricius } \\
{[43]}\end{array}$ \\
\hline Porites cylindrica (Dana, 1821) & 1-30mg/I SPM (weeks) & $\begin{array}{c}\text { Skeletal growth sustained, } \\
\text { tissue biomass decreased at } \\
\text { high SPM }\end{array}$ & M & 1.5 & $\begin{array}{c}\text { Anthony \& Fabricius } \\
{[43]}\end{array}$ \\
\hline Porites cylindrica (Dana, 1821) & 1-30mg/I SPM (hours) & $\begin{array}{l}\text { Increased feeding capacity at } \\
\text { high SPM concentrations }\end{array}$ & M & 1.5 & Anthony [30] \\
\hline Porites cylindrica (Dana, 1821) & $\begin{array}{l}\text { 1-16mg/I suspended } \\
\text { matter ( } 8 \text { weeks) }\end{array}$ & No effect on growth rates & M & 1.5 & Anthony [44] \\
\hline Porites cylindrica (Dana, 1821) & $\begin{array}{l}\text { Shading (equiv. } \\
16 \mathrm{mg} / \mathrm{I} \text { at } 4 \mathrm{~m})(8 \\
\text { weeks) }\end{array}$ & $\begin{array}{l}\text { Significant reduction in } \\
\text { growth rate }\end{array}$ & M & 1.5 & Anthony [44] \\
\hline $\begin{array}{l}\text { Porites divaricate (Lesueur, } \\
\text { 1821) }\end{array}$ & $\begin{array}{l}50-150-476 \mathrm{mg} / \mathrm{l}(96 \\
\mathrm{h})\end{array}$ & $\begin{array}{l}\text { No effect at } 50 \text { and } 150 \mathrm{mg} / \mathrm{l} \\
\text { extreme sublethal stress (but } \\
\text { survival) at } 476 \mathrm{mg} / \mathrm{I}\end{array}$ & B & 1.2 & Thompson [24] \\
\hline $\begin{array}{l}\text { Porites divaricate (Lesueur, } \\
\text { 1821) }\end{array}$ & $1000 \mathrm{mg} / \mathrm{I}($ for $65 \mathrm{~h}$ ) & No mortality & B & 1.2 & $\begin{array}{c}\text { Thompson \& Bright } \\
{[28]}\end{array}$ \\
\hline Porites utcata (Lamarck, 1816) & $\begin{array}{l}50-150-476 \mathrm{mg} / \mathrm{I}(96 \\
\mathrm{h})\end{array}$ & $\begin{array}{l}\text { No effect at } 50 \text { and } 150 \mathrm{mg} / \mathrm{l} \\
\text { extreme sublethal stress (but } \\
\text { survival) at } 476 \mathrm{mg} / \mathrm{I}\end{array}$ & B & 2 & Thompson [24] \\
\hline Porites utcata (Lamarck, 1816) & $1000 \mathrm{mg} / \mathrm{l}$ (for $65 \mathrm{~h}$ ) & No mortality & B & 2 & $\begin{array}{c}\text { Thompson \& Bright } \\
{[28]}\end{array}$ \\
\hline Porites lobata (Dana, 1846) & - & Dominant in turbid waters & - & - & Stafford-Smith [48] \\
\hline $\begin{array}{c}\text { Porites lutea (Milne Edwards } \\
\text { \&Haime, 1851) }\end{array}$ & - & Dominant in turbid waters & M & 1.5 & Stafford-Smith [48] \\
\hline $\begin{array}{l}\text { Porites lutea (Milne Edwards } \\
\text { \&Haime, 1851) }\end{array}$ & $\begin{array}{l}\text { Increased turbidity up } \\
\text { to } 286 \mathrm{mg} / \mathrm{l} \text { ( } 4 \text { months) }\end{array}$ & $\begin{array}{l}\text { Partial mortality of } 25 \% \text { of } \\
\text { colonies, recovery within } 22 \\
\text { months }\end{array}$ & M & 1.5 & Brown et al. [53] \\
\hline Porites (Pallas, 1766) & $\begin{array}{l}\text { Significant light } \\
\text { reduction due to } \\
\text { eutrophication }\end{array}$ & $\begin{array}{c}\text { Reduced reproductive success } \\
\text { (ova maturation, larval } \\
\text { development) }\end{array}$ & M & 2 & Tomascik \& Sander [54] \\
\hline Porites sp. & $\begin{array}{c}\text { General increase in } \\
\text { SPM }\end{array}$ & $\begin{array}{l}\text { Decreasing tissue thickness } \\
\text { from nearshore to offshore }\end{array}$ & - & - & Barnes \& Lough [55] \\
\hline Porites sp. & $\begin{array}{c}\text { General increase in } \\
\text { SPM }\end{array}$ & $\begin{array}{c}\text { Decreasing skeletal density, } \\
\text { linear extension, increasing } \\
\text { calcification }\end{array}$ & - & - & Lough \& Barnes $[56,57]$ \\
\hline $\begin{array}{l}\text { Sarcophytonglaucum } \\
\text { (Quoy\&Gaimard, 1833) }\end{array}$ & $\begin{array}{l}\text { Light reduced to } 50 \% \\
\text { and } 25 \% \text { PAR (surface) }\end{array}$ & $\begin{array}{c}\text { Severely diminished } \\
\text { productivity, increased carbon } \\
\text { loss and mucus }\end{array}$ & So & - & Riegl \& Branch [39] \\
\hline
\end{tabular}




\begin{tabular}{|c|c|c|c|c|c|}
\hline Coral Species & $\begin{array}{l}\text { Turbidity Level } \\
\text { (Tested) }\end{array}$ & Response & Growth Form & $\begin{array}{l}\text { Calyx } \\
(\mathrm{Mm})\end{array}$ & References \\
\hline $\begin{array}{l}\text { Scolymiacubensis (Milne } \\
\text { Edwards \&Haime, 1849) }\end{array}$ & $\begin{array}{c}\text { 49,101,165 and } \\
199 \mathrm{mg} / \mathrm{l}(10-20 \text { days })\end{array}$ & $\begin{array}{l}\text { No effect on growth rate or } \\
\text { survival after } 10 \mathrm{~d} \text {, minor } \\
\text { bleaching after } 20 \mathrm{~d}\end{array}$ & S & 91 & Rice \& Hunter [37] \\
\hline $\begin{array}{l}\text { Scolymiacubensis (Milne } \\
\text { Edwards \&Haime, 1849) }\end{array}$ & 49-199mg/l (10 days) & $\begin{array}{l}\text { Partial polyp death and } \\
\text { partial bleaching (in some } \\
\text { indivduals) }\end{array}$ & S & 91 & Rice [58] \\
\hline $\begin{array}{c}\text { Siderastrea radians (Pallas, } \\
1766 \text { ) }\end{array}$ & 49-199mg/l (10 days) & $\begin{array}{l}\text { Partial polyp death and } \\
\text { partial bleaching (in some } \\
\text { indivduals) }\end{array}$ & $\mathrm{M} / \mathrm{E}$ & 5 & Rice [58] \\
\hline $\begin{array}{l}\text { Siderastreasiderea (Ellis \& So/ } \\
\text { ander, 1786) }\end{array}$ & $\begin{array}{l}\text { Severe light reduction } \\
\text { (shading) for } 5 \text { weeks }\end{array}$ & $\begin{array}{c}\text { Partial bleaching after } 5 \\
\text { weeks, partial recovery in 6-8 } \\
\text { weeks }\end{array}$ & M & 3 & Rogers [23] \\
\hline Sinularia dura (Pratt, 1903) & $\begin{array}{l}\text { Light reduced to } 50 \% \\
\text { and } 25 \% \text { PAR (surface) }\end{array}$ & $\begin{array}{c}\text { Severely diminished } \\
\text { productivity, increased carbon } \\
\text { loss and mucus }\end{array}$ & So & - & Riegl \& Branch [39] \\
\hline $\begin{array}{l}\text { Sinularialeptoelados } \\
\text { (Ehrenberg, 1834) }\end{array}$ & $\begin{array}{l}\text { Light reduced to } 50 \% \\
\text { and } 25 \% \text { PAR (surface) }\end{array}$ & $\begin{array}{c}\text { Severely diminished } \\
\text { productivity, increased carbon } \\
\text { loss and mucus }\end{array}$ & So & - & Riegl \& Branch [39] \\
\hline Solenastrea hyades (Dana, 1846) & $\begin{array}{c}\text { 49,101,165 and } \\
199 \mathrm{mg} / \mathrm{l}(10-20 \text { days })\end{array}$ & $\begin{array}{l}\text { No effect on growth rate or } \\
\text { survival after } 10 \mathrm{~d} \text {, minor } \\
\text { bleaching after } 20 \mathrm{~d}\end{array}$ & M & 5 & Rice \& Hunter [37] \\
\hline Solenastrea hyades (Dana, 1846) & 49-199mg/l (10 days) & $\begin{array}{l}\text { Partial polyp death and } \\
\text { partial bleaching (in some } \\
\text { individuals) }\end{array}$ & M & 5 & Rice [58] \\
\hline $\begin{array}{l}\text { Stephanocoenia intersepta } \\
\text { (Lamarck,1816) }\end{array}$ & $\begin{array}{c}\text { 49,101,165 and } \\
199 \mathrm{mg} / \mathrm{l}(10-20 \text { days })\end{array}$ & $\begin{array}{l}\text { No effect on growth rate or } \\
\text { survival after } 10 \mathrm{~d} \text {, minor } \\
\text { bleaching after } 20 \mathrm{~d}\end{array}$ & M & 3 & Rice \& Hunter [37] \\
\hline $\begin{array}{l}\text { Stephanocoenia intersepea } \\
\text { (Lamarck, 1816) }\end{array}$ & 49-199mg/l (10 days) & $\begin{array}{l}\text { Partial polyp death and } \\
\text { partial bleaching (in some } \\
\text { individuals) }\end{array}$ & M & 3 & Rice [58] \\
\hline $\begin{array}{l}\text { Turbinaria mesenterina } \\
\text { (Lamarck, 1816) }\end{array}$ & - & Tolerant to high turbidity & $\mathrm{L}$ & 1.5 & Nieuwaal [25] \\
\hline $\begin{array}{l}\text { Turbinaria reniformis (Bernard, } \\
\text { 1896) }\end{array}$ & - & Tolerant to high turbidity & $\mathrm{L}$ & 2 & Nieuwaal [25] \\
\hline Turbinaria spp. & - & $\begin{array}{l}\text { Most tolerant to high turbidity } \\
\text { and sedimentation }\end{array}$ & & & $\begin{array}{c}\text { Stoddart \&Stoddart } \\
{[59]}\end{array}$ \\
\hline
\end{tabular}

Abbreviations used in the tables are B-Branching; C-Columnar; (including digitate); E-Encrusting; F-Foliaceous; L-Laminar (includingplate \& Tabular); M-Massive; S-Solitary (Free-living); So-Soft corals \& Gorgonians. Calyx diameter measured on museum specimens, supplemented with data from Stafford-Smith \& Ormond (1992).

Table 5: Quantitative coral response to sedimentation

\begin{tabular}{|c|c|c|c|c|c|}
\hline Coral species & $\begin{array}{l}\text { Sedimentation rate (test- } \\
\text { ed) }\end{array}$ & Response & Growth form & Calyx (mm) & References \\
\hline $\begin{array}{c}\text { Acropora cervicornis (La- } \\
\text { marck. 1816) }\end{array}$ & $\begin{array}{c}200 \mathrm{mg} / \mathrm{m}^{2} / \mathrm{d} \text { (daily for } 45 \\
\text { days) }\end{array}$ & $\begin{array}{c}\text { No effect (not even on } \\
\text { growth rate) even after } \\
45 \text { days }\end{array}$ & B & 1 & Rogers [23] \\
\hline $\begin{array}{l}\text { Acropora cervicornis (La- } \\
\text { marck. 1816) }\end{array}$ & $200 \mathrm{mg} \mathrm{cm}^{-2} \mathrm{~d}^{-1}$ (daily) & No effect & B & 1 & Rogers [21] \\
\hline $\begin{array}{c}\text { Acropora cervicornis (La- } \\
\text { marck, 1816) }\end{array}$ & $430 \mathrm{mg} \mathrm{cm}^{-2} \mathrm{~d}^{-1}$ (>1 day) & Physiological stress & B & 1 & $\begin{array}{c}\text { Bak \& Elgershuizen } \\
\text { [63] }\end{array}$ \\
\hline
\end{tabular}




\begin{tabular}{|c|c|c|c|c|c|}
\hline Coral species & $\begin{array}{c}\text { Sedimentation rate (test- } \\
\text { ed) }\end{array}$ & Response & Growth form & Calyx (mm) & References \\
\hline $\begin{array}{l}\text { Acropora cervicornis (La- } \\
\text { marck, 1816) }\end{array}$ & $\begin{array}{c}\text { Burial }(10-12 \mathrm{~cm} \text { of reef } \\
\text { sand })\end{array}$ & $\begin{array}{c}\text { Sublethal stress within } 12 \\
\text { h; } 100 \% \text { mortality within } \\
72 \mathrm{~h}\end{array}$ & B & 1 & Thompson [64] \\
\hline $\begin{array}{c}\text { Acropora formosa (Dana, } \\
1846 \text { ) }\end{array}$ & $\begin{array}{l}\text { Up to } 14.6 \mathrm{mg} / \mathrm{m}^{2} / \mathrm{d} \text { (fine } \\
\text { silt) due to dredging }\end{array}$ & $\begin{array}{l}\text { No effect on growth rate } \\
\text { (in situ) }\end{array}$ & B & 1.2 & Chansang et al. [65] \\
\hline $\begin{array}{c}\text { Acropora formosa (Dana, } \\
1846 \text { ) }\end{array}$ & $\begin{array}{c}200-300 \mathrm{mg} \mathrm{cm}^{-2} \mathrm{~d}^{-1} \text { (up to } \\
7 \text { days) }\end{array}$ & Decreased growth & B & 1.2 & Simpson [66] \\
\hline $\begin{array}{l}\text { Acropora millepora (Eh- } \\
\text { renberg, 1834) (larvae) }\end{array}$ & $0.5-325 \mathrm{mg} \mathrm{cm}^{-2} \mathrm{~d}^{-1}$ (2 days) & $\begin{array}{c}\text { Reduction of larval settle- } \\
\text { ment }\end{array}$ & - & - & Babcock [67] \\
\hline $\begin{array}{l}\text { Acropora millepora (Eh- } \\
\text { renberg, 1834) }\end{array}$ & $\begin{array}{c}83 \mathrm{mg} \mathrm{cm}^{-2} \mathrm{~d}^{-1} \text { (up to } 16 \\
\text { weeks) }\end{array}$ & $\begin{array}{c}\text { Onset mortality after } 4 \\
\text { weeks, full mortality after } \\
12 \text { weeks }\end{array}$ & B & 1 & $\begin{array}{l}\text { Negri et al. [32] \& } \\
\text { Flores et al. [33] }\end{array}$ \\
\hline $\begin{array}{l}\text { Acropora palifera (La- } \\
\text { marck 1816) }\end{array}$ & $\begin{array}{l}\text { Field site comparison }(<1 \\
\left.\text { versus } 13.5 \mathrm{mg} \mathrm{cm}^{-2} \mathrm{~d}^{-1}\right)\end{array}$ & $\begin{array}{l}\text { Reduced fecundity at the } \\
\text { site with higher sedimen- } \\
\text { tation }\end{array}$ & $\mathrm{L}$ & 2 & Kojis \&Quinn [68] \\
\hline $\begin{array}{l}\text { Acropora palmata (La- } \\
\text { marck, 1816) }\end{array}$ & $\begin{array}{l}\text { Up to } 600 \mathrm{mg} \mathrm{cm}^{-2} \mathrm{~d}^{-1} \text { (natu- } \\
\text { ral events) }\end{array}$ & $\begin{array}{l}\text { Poor rejection ability; } \\
\text { sediment accumulation }\end{array}$ & B & 2 & $\begin{array}{c}\text { Abdel-Salam \& Porter } \\
{[69]}\end{array}$ \\
\hline $\begin{array}{l}\text { Acropora palmata (La- } \\
\text { marck. 1816) }\end{array}$ & $430 \mathrm{mg} \mathrm{cm}^{-2} \mathrm{~d}^{-1}$ (>1 day) & Physiological stress & B & 2 & $\begin{array}{c}\text { Bak \& Elgershuizen } \\
{[63]}\end{array}$ \\
\hline $\begin{array}{l}\text { Acroporo palmata (La- } \\
\text { marck, 1816) }\end{array}$ & $200 \mathrm{mg} \mathrm{cm}^{-2} \mathrm{~d}^{-1}$ (once) & Partial mortality & B & 2 & Rogers [70] \\
\hline $\begin{array}{l}\text { Acropora palmata (La- } \\
\text { marck, 1816) }\end{array}$ & $\begin{array}{c}200 \mathrm{mg} \mathrm{cm}^{-2} \mathrm{~d}^{-1} \text { (field appli- } \\
\text { cation) }\end{array}$ & Death of underlying tissue & B & 2 & Rogers [21] \\
\hline
\end{tabular}




\begin{tabular}{|c|c|c|c|c|c|}
\hline Coral species & $\begin{array}{l}\text { Sedimentation rate (test- } \\
\text { ed) }\end{array}$ & Response & Growth form & Calyx (mm) & References \\
\hline $\begin{array}{l}\text { Acropora palmata (La- } \\
\text { marck. 1816) }\end{array}$ & $\begin{array}{c}\text { Burial }(10-12 \mathrm{~cm} \text { of reef } \\
\text { sand })\end{array}$ & $\begin{array}{l}\text { 100\% mortality within } \\
72 \mathrm{~h}\end{array}$ & B & 2 & Thompson [64] \\
\hline Acropora sp. & $5 \mathrm{mg} \mathrm{cm}^{-2} \mathrm{~d}^{-1}$ & $\begin{array}{l}\text { Massive mucus production } \\
\text { (within } 1 \mathrm{~h} \text { ). Sublethal }\end{array}$ & - & - & $\begin{array}{c}\text { Fabricius \& Wolanski } \\
\qquad 35]\end{array}$ \\
\hline Acropora sp. & Burial for $20 \mathrm{~h}$ & Mortality of all colonies & - & - & Wesseling et al. [71] \\
\hline Acropora sp. & $39.6 \mathrm{mg} \mathrm{cm}^{-2} \mathrm{~d}^{-1}$ (for 2 weeks) & $\begin{array}{l}\text { Partial bleaching (less } \\
\text { affected) }\end{array}$ & - & - & Fabricius et al. [72] \\
\hline $\begin{array}{l}\text { Agaricia agaricites (Linnae- } \\
\text { us, 1758) }\end{array}$ & $\begin{array}{l}\text { Heavy sedimentation event } \\
\qquad(>1 \mathrm{~cm})\end{array}$ & $\begin{array}{l}\text { Reduced growth but } \\
\text { survival }\end{array}$ & $\mathrm{L}$ & 5 & Bak [36] \\
\hline $\begin{array}{c}\text { Agaricia agaricites (Linnae- } \\
\text { us, 1758) }\end{array}$ & $430 \mathrm{mg} \mathrm{cm}^{-2} \mathrm{~d}^{-1}$ (sand) & Mortality after 1 day & L & 5 & $\begin{array}{c}\text { Bak \& Elgershuizen } \\
{[63]}\end{array}$ \\
\hline $\begin{array}{l}\text { Agaricia agaricites (Linnae- } \\
\text { us, 1758) }\end{array}$ & $\begin{array}{c}\text { Burial }(10-12 \mathrm{~cm} \text { of reef } \\
\text { sand })\end{array}$ & $\begin{array}{c}\text { 60\% Tissue loss within } 24 \\
\text { h; } 100 \% \text { mortality after } \\
72 \mathrm{~h}\end{array}$ & $\mathrm{~L}$ & 5 & Thompson [64] \\
\hline $\begin{array}{l}\text { Agaricia lamarcki (Milne } \\
\text { Edwards \& Haime, 1851) }\end{array}$ & $\begin{array}{c}140 \mathrm{mg} / \mathrm{m}^{2} / \mathrm{d} \text { (mean) for } \\
\text { several weeks }\end{array}$ & $\begin{array}{l}\text { Mass mortality ( } 4 \text { years } \\
\text { after the steep decline in } \\
\text { growth) }\end{array}$ & $\mathrm{L}$ & 8 & Van ‘t Hof [73] \\
\hline Agaricia sp. & $30 \mathrm{mg} / \mathrm{m}^{2} / \mathrm{d}$ (natural) & $\begin{array}{l}\text { No effect: dominant } \\
\text { species }\end{array}$ & - & - & Loya [74] \\
\hline Atveopora sp. & - & $\begin{array}{l}\text { Can survive high sedimen- } \\
\text { tation rates }\end{array}$ & - & - & $\begin{array}{l}\text { Stafford-Smith \& } \\
\text { Ormond [75] }\end{array}$ \\
\hline $\begin{array}{l}\text { Astrangia poculata (Ellis } \\
\text { Solander, 1786) }\end{array}$ & $<600 \mathrm{mg} \mathrm{cm}^{-2} \mathrm{~d}^{1}$ & Survival & $S$ & 6 & Peters \& Pilson [76] \\
\hline
\end{tabular}




\begin{tabular}{|c|c|c|c|c|c|}
\hline Coral species & $\begin{array}{c}\text { Sedimentation rate (test- } \\
\text { ed) }\end{array}$ & Response & Growth form & Calyx (mm) & References \\
\hline $\begin{array}{l}\text { Catalaphyllia jardinei } \\
\text { (Saville-Kent, 1893) }\end{array}$ & - & $\begin{array}{l}\text { Survive high sedimenta- } \\
\text { tion rates }\end{array}$ & M & 40 & $\begin{array}{l}\text { Stafford-Smith \& } \\
\text { Ormond [75] }\end{array}$ \\
\hline $\begin{array}{l}\text { Cladocora arbuscula (Le- } \\
\text { sueur, 1812) }\end{array}$ & Complete burial & $50 \%$ Survival after 15 days & B & 4 & Rice \& Hunter [37] \\
\hline $\begin{array}{l}\text { Ctenactis echinata (Pallas, } \\
\text { 1766) }\end{array}$ & $\begin{array}{l}\text { Continuously repeated burial } \\
\text { (sand) }\end{array}$ & $\begin{array}{l}\text { Tissue mortality and colo- } \\
\text { ny death after } 24-72 \mathrm{~h}\end{array}$ & $S$ & 200 & Schuhmacher [77] \\
\hline $\begin{array}{l}\text { Cycloseris costulata (Ort- } \\
\text { mann, 1889) }\end{array}$ & $\begin{array}{l}\text { Continuously repeated burial } \\
\text { (sand) }\end{array}$ & $\begin{array}{l}\text { Survival (endurance with } \\
\text { no apparent effect) }\end{array}$ & S & 15 & Schuhmacher [77] \\
\hline $\begin{array}{l}\text { Cycloseris costulata (Ortr- } \\
\text { nann, 1889) }\end{array}$ & $40 \mathrm{~mm}^{3} / \mathrm{cm}^{2} / \mathrm{d}$ & $\begin{array}{l}\text { Maximum rate tolerated } \\
\text { (field gradient) }\end{array}$ & S & 15 & Schuhmacher [77] \\
\hline $\begin{array}{l}\text { Cycloseris distorta (Miche- } \\
\text { lin, 1842) }\end{array}$ & - & $\begin{array}{l}\text { Efficient sediment rejector } \\
\text { (polyp inflation) }\end{array}$ & S & 7.5 & Schuhmacher [77] \\
\hline Cycloseris spp. & - & $\begin{array}{l}\text { Can actively dig through } \\
\text { overlying sediment }\end{array}$ & - & - & $\begin{array}{l}\text { Stafford-Smith \& } \\
\text { Ormond [75] }\end{array}$ \\
\hline $\begin{array}{l}\text { Danafungta horrida (Dana, } \\
1846 \text { ) }\end{array}$ & $\begin{array}{l}\text { Continuously repeated burial } \\
\text { (sand) }\end{array}$ & $\begin{array}{l}\text { Tissue mortality and colo- } \\
\text { ny death after } 24-72 \mathrm{~h}\end{array}$ & S & 215 & Schuhmacher [77] \\
\hline $\begin{array}{l}\text { Danajungia scruposa } \\
\text { (Klunzinger, 1879) }\end{array}$ & $\begin{array}{l}\text { Continuously repeated burial } \\
\text { (sand) }\end{array}$ & $\begin{array}{l}\text { Tissue mortality and colo- } \\
\text { ny death after } 24-72 \mathrm{~h}\end{array}$ & S & 380 & Schuhmacher [77] \\
\hline $\begin{array}{l}\text { Dichococnia stokes (Milne } \\
\text { Edwards \&Haime, 1848) }\end{array}$ & $430 \mathrm{mg} \mathrm{cm}^{-2} \mathrm{~d}^{1}($ sand + oil $)$ & Mortality after 1 day & M & 11 & $\begin{array}{c}\text { Bak \& Elgershuizen } \\
\text { [63] }\end{array}$ \\
\hline
\end{tabular}




\begin{tabular}{|c|c|c|c|c|c|}
\hline Coral species & $\begin{array}{l}\text { Sedimentation rate (test- } \\
\text { ed) }\end{array}$ & Response & Growth form & Calyx (mm) & References \\
\hline $\begin{array}{l}\text { Diploastrea ellopora (La- } \\
\text { marck, 1816) }\end{array}$ & $20 \mathrm{mg} \mathrm{cm}^{-2} \mathrm{~d}^{1}$ (mixed sand) & Survival (4 months) & M & 14 & Todd et al. [78] \\
\hline $\begin{array}{l}\text { Diporia clivosa (Ellis \& } \\
\quad \text { Solander, 1786) }\end{array}$ & $\begin{array}{l}\text { Repeated application of } 200 \\
\mathrm{mg} / \mathrm{cm}^{2}\end{array}$ & Extensive damage & M & 9 & Rogers [79] \\
\hline $\begin{array}{l}\text { Diploria labyrInthiformis } \\
\text { (Linnaeus, 1758) }\end{array}$ & $\begin{array}{l}\text { High sedimentation rates } \\
\text { (dredging) }\end{array}$ & Survival (no effect) & M & 8 & Dodge \& Vai snys [80] \\
\hline $\begin{array}{c}\text { Diploria strigosa (Dana, } \\
1846)\end{array}$ & $\begin{array}{l}\text { Up to } 600 \mathrm{mg} \mathrm{cm}^{-2} \mathrm{~d}^{-1} \text { (natu- } \\
\text { ral events) }\end{array}$ & $\begin{array}{l}\text { High sediment clearing } \\
\text { rate }\end{array}$ & M & 8 & $\begin{array}{c}\text { Abdel-Salam \& Porter } \\
{[69]}\end{array}$ \\
\hline $\begin{array}{c}\text { Diploria strigosa (Dana, } \\
1846 \text { ) }\end{array}$ & $200 \mathrm{mg} \mathrm{cm}^{-2} \mathrm{~d}^{1}$ (daily) & No effect & M & 8 & Rogers [21] \\
\hline $\begin{array}{c}\text { Diploria strtgosa (Dana, } \\
1846 \text { ) }\end{array}$ & $\begin{array}{l}\text { High sedimentation rates } \\
\text { (dredging) }\end{array}$ & $\begin{array}{c}\text { Mass mortality ( } 4 \text { years } \\
\text { after the steep decline in } \\
\text { growth) }\end{array}$ & M & 8 & Dodge \& Vaisnys [80] \\
\hline $\begin{array}{c}\text { Diploria strigosa (Dana, } \\
1846 \text { ) }\end{array}$ & $\begin{array}{l}\text { Burial }(10-12 \mathrm{~cm} \text { of reef } \\
\text { sand })\end{array}$ & $\begin{array}{l}\text { Partial bleaching and sub } \\
\text { lethal stress within } 24 \mathrm{~h}\end{array}$ & M & 8 & Thompson [64] \\
\hline $\begin{array}{l}\text { Duncanopsammia axifuga } \\
\text { (Milne Edwards \& Halme. } \\
\text { 1848) }\end{array}$ & - & $\begin{array}{l}\text { Can survive high sedimen- } \\
\text { tation rates }\end{array}$ & B & 14 & $\begin{array}{l}\text { Stafford-Smith \& } \\
\text { Ormond [75] }\end{array}$ \\
\hline Ecitinopora spp. & - & Active sediment rejecter & - & - & $\begin{array}{l}\text { Stafford-Smith \& } \\
\text { Ormond [75] }\end{array}$ \\
\hline $\begin{array}{l}\text { Echinopora mammlformis } \\
\text { (Nemenzo, 1959) }\end{array}$ & - & Active sediment rejecter & L & 5 & $\begin{array}{l}\text { Stafford-Smith \& } \\
\text { Ormond [75] }\end{array}$ \\
\hline
\end{tabular}




\begin{tabular}{|c|c|c|c|c|c|}
\hline Coral species & $\begin{array}{l}\text { Sedimentation rate (test- } \\
\text { ed) }\end{array}$ & Response & Growth form & Calyx (mm) & References \\
\hline Euphyllia spp. & - & $\begin{array}{l}\text { Can survive high sedimen- } \\
\text { tation rates }\end{array}$ & - & - & $\begin{array}{l}\text { Stafford-Smith \& } \\
\text { Ormond [75] }\end{array}$ \\
\hline $\begin{array}{c}\text { Favia favus } \\
\text { (Forskal, 1775) }\end{array}$ & $200 \mathrm{mg} \mathrm{cm}^{-2} \mathrm{~d}^{-1}$ ( 6 weeks) & $\begin{array}{l}\text { Minor tissue damage, } \\
\text { mucus production, no } \\
\text { bleaching }\end{array}$ & M & 14,0 & $\begin{array}{c}\text { Riegl [81] \& Bloomer } \\
\text { [82] }\end{array}$ \\
\hline $\begin{array}{l}\text { Favia speciosa } \\
\text { (Dana, 1846) }\end{array}$ & $20 \mathrm{mg} \mathrm{cm}^{-2} \mathrm{~d}^{-1}$ (mixed sand) & Survival (4 months) & M & 12 & Todd et al. [78] \\
\hline Favia sp. & $\left(0.9-1.3 \mathrm{mg} / \mathrm{m}^{2} /\right.$ day $)$ & $\begin{array}{l}\text { Described as relatively } \\
\text { 'sensitive' to sedimenta- } \\
\text { tion }\end{array}$ & - & - & $\begin{array}{c}\text { McClanahan \& Obura } \\
{[93]}\end{array}$ \\
\hline $\begin{array}{l}\text { Favia stelligera (Dana, } \\
\text { 1846) }\end{array}$ & $200 \mathrm{mg} \mathrm{cm}^{-2} \mathrm{~d}^{-1}$ & Mortality within 1-2 days & M & 6 & Stafford-Smith [48] \\
\hline $\begin{array}{l}\text { Favites pentagona (Esper, } \\
1794)\end{array}$ & $200 \mathrm{mg} \mathrm{cm}^{-2} \mathrm{~d}^{-1}$ ( 6 weeks) & $\begin{array}{l}\text { Tissue damage, mucus } \\
\text { production }\end{array}$ & M & 7 & $\begin{array}{c}\text { Riegl [81] \& Bloomer } \\
{[82]}\end{array}$ \\
\hline Favites spp. & $\begin{array}{l}\text { (between } 1.3 \text { and } 4 \mathrm{mg} \\
\mathrm{cm}^{-2} \mathrm{~d}^{-1} \text { ) }\end{array}$ & $\begin{array}{c}\text { Tolerance to sedimenta- } \\
\text { tion described as 'inter- } \\
\text { mediate.' }\end{array}$ & - & - & $\begin{array}{c}\text { McClanahan \& Obura } \\
{[83]}\end{array}$ \\
\hline $\begin{array}{l}\text { Fungia fungites (Linnaeus, } \\
\text { 1758) }\end{array}$ & $\begin{array}{l}\text { Continuously repeated burial } \\
\text { (sand) }\end{array}$ & $\begin{array}{l}\text { Tissue mortality and colo- } \\
\text { ny death after } 24-72 \mathrm{~h}\end{array}$ & $S$ & 310 & Schuhmacher [77] \\
\hline $\begin{array}{l}\text { Fungia fungites (Linnaeus, } \\
\text { 1758) }\end{array}$ & $10 \mathrm{~mm}^{3} / \mathrm{cm}^{2} / \mathrm{d}$ & Maximum rate tolerated & S & 310 & Schuhmacher [77] \\
\hline $\begin{array}{c}\text { Galaxea fascicularis (Lin- } \\
\text { naeus, 1767) }\end{array}$ & $39.6 \mathrm{mg} \mathrm{cm}^{-2}$ (for 2 weeks) & $\begin{array}{l}\text { Sublethal (sed.accum.), act } \\
\text { removal (polyp), recovery }\end{array}$ & M & 8 & Fabricius et al. [72] \\
\hline
\end{tabular}




\begin{tabular}{|c|c|c|c|c|c|}
\hline Coral species & $\begin{array}{c}\text { Sedimentation rate (test- } \\
\text { ed) }\end{array}$ & Response & Growth form & Calyx (mm) & References \\
\hline $\begin{array}{l}\text { Galaxea fascicularis (Lin- } \\
\text { naeus, } 1767 \text { ) }\end{array}$ & Burial for $20 \mathrm{~h}$ & $\begin{array}{l}\text { Tissue bleaching, recovery } \\
\text { after } 4 \text { weeks }\end{array}$ & M & 8 & Wesseling et al. [71] \\
\hline Galaxea spp. & $\left(4 \mathrm{mg} / \mathrm{m}^{2} /\right.$ day) & $\begin{array}{l}\text { Tolerance to sedimenta- } \\
\text { tion described as 'inter- } \\
\text { mediate' }\end{array}$ & - & - & $\begin{array}{c}\text { McClanahan \& Obura } \\
{[83]}\end{array}$ \\
\hline $\begin{array}{l}\text { Gardineroseris planulata } \\
\text { (Dana, 1846) }\end{array}$ & $200 \mathrm{mg} \mathrm{cm}^{-2} \mathrm{~d}^{-1}$ & $\begin{array}{c}\text { Partial mortality after } 6 \\
\text { days }\end{array}$ & M & 7 & Stafford-Smith [48] \\
\hline $\begin{array}{l}\text { Goniastrea retiformis } \\
\quad \text { (Lamark, 1816) }\end{array}$ & - & $\begin{array}{l}\text { Common on reefs affected } \\
\text { by sedimentation }\end{array}$ & M & 4 & $\begin{array}{c}\text { Brown \& Howard } \\
{[84]}\end{array}$ \\
\hline $\begin{array}{l}\text { Goniopora lobate (Milne } \\
\text { Edwards \& Haime, 1860) }\end{array}$ & - & Active sediment rejecter & $\mathrm{s}$ & 4 & $\begin{array}{l}\text { Stafford-Smith \& } \\
\text { Ormond [75] }\end{array}$ \\
\hline Goniopora spp. & - & $\begin{array}{l}\text { Survive high sedimenta- } \\
\text { tion rates }\end{array}$ & - & - & $\begin{array}{l}\text { Stafford-Smith \& } \\
\text { Ormond [75] }\end{array}$ \\
\hline $\begin{array}{l}\text { Gyrosmilia nterrupta } \\
\text { (Ehrenberg,1834) }\end{array}$ & $200 \mathrm{mg} \mathrm{cm}^{-2} \mathrm{~d}^{-1}$ (6 weeks) & $\begin{array}{l}\text { Tissue damage, mucus pro- } \\
\text { duction, no bleaching }\end{array}$ & $\mathrm{M} / \mathrm{E}$ & 16 & $\begin{array}{l}\text { Riegl [81] \& Riegl and } \\
\text { Bloomer [82] }\end{array}$ \\
\hline $\begin{array}{l}\text { Heliofungia actiniformis } \\
\text { (Quoy \& Gaimard, 1833) }\end{array}$ & - & $\begin{array}{l}\text { Efficient sediment rejecter } \\
\text { (polyp inflation) }\end{array}$ & S & 210 & Schuhmacher [77] \\
\hline $\begin{array}{l}\text { Helioporu coerulea (Pallas, } \\
\text { 1766) }\end{array}$ & Burial for $20 \mathrm{~h}$ & $\begin{array}{c}\text { Tissue bleaching, recovery } \\
\text { after } 4 \text { weeks }\end{array}$ & B & 0.8 & Wesseling et al. [71] \\
\hline $\begin{array}{l}\text { Heteropsammia cochlea } \\
\text { (Spengler, 1783) }\end{array}$ & - & $\begin{array}{l}\text { Obligate commensal sipun- } \\
\text { culid prevents burial }\end{array}$ & $S$ & 7 & $\begin{array}{l}\text { Stafford-Smith \& } \\
\text { Ormond [75] }\end{array}$ \\
\hline
\end{tabular}




\begin{tabular}{|c|c|c|c|c|c|}
\hline Coral species & $\begin{array}{l}\text { Sedimentation rate (test- } \\
\text { ed) }\end{array}$ & Response & Growth form & Calyx (mm) & References \\
\hline Hydnophora spp. & $\left(4 \mathrm{mg} / \mathrm{m}^{2} /\right.$ day) & $\begin{array}{l}\text { Tolerance to sedimenta- } \\
\text { tion described as 'inter- } \\
\text { mediate.' }\end{array}$ & - & - & $\begin{array}{c}\text { McClanahan \& Obura } \\
\text { [83] }\end{array}$ \\
\hline $\begin{array}{c}\text { Isopora polifera (Lamarck, } \\
\text { 1816) }\end{array}$ & $10-15 \mathrm{mg} \mathrm{cm}^{-2} \mathrm{~d}^{-1}$ & $\begin{array}{l}50 \% \text { Reduction in fecun- } \\
\text { dity }\end{array}$ & $\mathrm{C}$ & 2 & Kojis \& Quinn [68] \\
\hline $\begin{array}{l}\text { Isophyllia sinuous (Ellis \& } \\
\text { Solander, 1786) }\end{array}$ & Complete burial & $\begin{array}{c}50 \% \text { Survival after } 7.2 \\
\text { days }\end{array}$ & M & 15 & Rice \& Hunter [37] \\
\hline $\begin{array}{l}\text { Leptoria phrygia (Ellis \& } \\
\text { Solander, 1786) }\end{array}$ & $25 \mathrm{mg} \mathrm{cm}^{-2} \mathrm{~d}^{-1}$ & $\begin{array}{l}\text { Minor tissue damage with- } \\
\text { in } 3 \text { weeks }\end{array}$ & - & 4.1 & Stafford-Smith [85] \\
\hline $\begin{array}{l}\text { Leptoria phrygia (Ellis \& } \\
\text { Solander, 1786) }\end{array}$ & $50-100 \mathrm{mg} \mathrm{cm}^{-2} \mathrm{~d}^{-1}$ & $\begin{array}{l}\text { Major tissue damage and } \\
\text { bleaching after } 4 \text { days }\end{array}$ & M & 4.1 & Stafford-Smith [85] \\
\hline $\begin{array}{l}\text { Leptoria phrygia (Ellis \& } \\
\text { Solander, 1786) }\end{array}$ & $100-200 \mathrm{mg} \mathrm{cm}^{-2} \mathrm{~d}^{-1}$ & $\begin{array}{l}\text { Partial mortality and } \\
\text { bleaching after } 4 \text { days }\end{array}$ & M & 4.1 & Stafford-Smith [85] \\
\hline $\begin{array}{l}\text { Leptoria phlygia (Ellis \& } \\
\text { Solander, 1786) }\end{array}$ & $>200 \mathrm{mg} \mathrm{cm}^{-2} \mathrm{~d}^{-1}$ & Mortality within 1-2 days & M & 4.1 & $\begin{array}{c}\text { Stafford-Smith [85], } \\
\text { [48] }\end{array}$ \\
\hline $\begin{array}{l}\text { Lobophytum depressum } \\
\text { (Tixier-Durivault, 1966) }\end{array}$ & $200 \mathrm{mg} \mathrm{cm}^{-2} \mathrm{~d}^{-1}$ ( 6 weeks) & $\begin{array}{l}\text { Tissue damage, bleaching, } \\
\text { and partial mortality }\end{array}$ & So & - & $\begin{array}{l}\text { Riegl [81] \& Riegl \& } \\
\text { Bloomer [82] }\end{array}$ \\
\hline $\begin{array}{l}\text { Lobophytum venustum } \\
\text { (Tixier-Durivault, 1957) }\end{array}$ & $200 \mathrm{mg} \mathrm{cm}^{-2} \mathrm{~d}^{-1}$ (6 weeks) & $\begin{array}{l}\text { Minor tissue damage and } \\
\text { bleaching }\end{array}$ & So & - & $\begin{array}{l}\text { Riegl [81] \& Riegl \& } \\
\quad \text { Bloomer [82] }\end{array}$ \\
\hline $\begin{array}{l}\text { Madracis auretenra (Locke } \\
\text {,Well \& Coates, 2007) }\end{array}$ & $\begin{array}{l}\text { Heavy sedimentation event } \\
\qquad(>1 \mathrm{~cm})\end{array}$ & $\begin{array}{l}\text { Reduced growth but } \\
\text { survival }\end{array}$ & B & 1 & Bak [36] \\
\hline
\end{tabular}




\begin{tabular}{|c|c|c|c|c|c|}
\hline Coral species & $\begin{array}{l}\text { Sedimentation rate (test- } \\
\text { ed) }\end{array}$ & Response & Growth form & Calyx (mm) & References \\
\hline $\begin{array}{c}\text { Manicina areolata (Lin- } \\
\text { neaus, 1758) }\end{array}$ & Complete burial & $50 \%$ Survival after 10 days & M & 23 & Rice \&Hunter [37] \\
\hline $\begin{array}{l}\text { Meandrina meandrites } \\
\text { (Linnaeus, 1758) }\end{array}$ & - & $\begin{array}{l}\text { Produces copious amounts } \\
\text { of mucus to remove silt }\end{array}$ & M & 15 & $\begin{array}{c}\text { Dumas \& Thomassin } \\
{[86]}\end{array}$ \\
\hline Millepora spp. & $\left(4 \mathrm{mg} / \mathrm{m}^{2} /\right.$ day $)$ & $\begin{array}{l}\text { Tolerance to sedimenta- } \\
\text { tion described as 'inter- } \\
\text { mediate.' }\end{array}$ & - & - & $\begin{array}{c}\text { McClanahan \& Obura } \\
\text { [83] }\end{array}$ \\
\hline $\begin{array}{l}\text { Montastraea annularis } \\
\text { (Ellis \& Solander, 1786) }\end{array}$ & - & $\begin{array}{l}\text { High sediment clearing } \\
\text { rate }\end{array}$ & $\mathrm{M} / \mathrm{E}$ & 5 & $\begin{array}{c}\text { Abdel-Salam \& Porter } \\
{[69]}\end{array}$ \\
\hline $\begin{array}{l}\text { Montastraea annularis } \\
\text { (Ellis \& Solander, 1786) }\end{array}$ & $\begin{array}{c}200 \mathrm{mg} \mathrm{cm}^{-2} \mathrm{~d}^{-1} \text { (daily appli- } \\
\text { cations) }\end{array}$ & $\begin{array}{c}\text { Tolerant for at least } 38 \\
\text { days }\end{array}$ & $\mathrm{L} / \mathrm{E}$ & 5 & Rogers [23] \\
\hline $\begin{array}{l}\text { Montastraea annularis } \\
\text { (Ellis \& Solander, 1786) }\end{array}$ & $\begin{array}{l}400-800 \mathrm{mg} \mathrm{cm}^{-2} \mathrm{~d}^{-1} \text { (single } \\
\text { application) }\end{array}$ & Mortality & M & 5 & Rogers [23] \\
\hline $\begin{array}{l}\text { Montastraea annularis } \\
\text { (Ellis \& Solander, 1786) }\end{array}$ & $19 \mathrm{mg} \mathrm{cm}^{-2} \mathrm{~d}^{-1}$ (permanent) & Reduced growth rate & $\mathrm{M} / \mathrm{E}$ & 5 & Torres [87] \\
\hline $\begin{array}{l}\text { Montastraea annufaris } \\
\text { (Ellis \& Solander, 1786) }\end{array}$ & $200 \mathrm{mg} \mathrm{cm}^{-2} \mathrm{~d}^{-1}$ (daily) & No effect & $\mathrm{M} / \mathrm{E}$ & 5 & Rogers [21] \\
\hline $\begin{array}{l}\text { Montastraea annuiaris } \\
\text { (Ellis \& Sotander, 1786) }\end{array}$ & $400 \mathrm{mg} \mathrm{cm}^{-2} \mathrm{~d}^{-1}$ & Temporary bleaching & $\mathrm{M} / \mathrm{E}$ & 5 & Rogers [21] \\
\hline
\end{tabular}




\begin{tabular}{|c|c|c|c|c|c|}
\hline Coral species & $\begin{array}{l}\text { Sedimentation rate (test- } \\
\text { ed) }\end{array}$ & Response & Growth form & Calyx (mm) & References \\
\hline $\begin{array}{l}\text { Montastraea annufaris } \\
\text { (Ellis \& Solander, 1786) }\end{array}$ & $800 \mathrm{mg} \mathrm{cm}^{-2} \mathrm{~d}^{-1}$ & Death of underlying tissue & $\mathrm{M} / \mathrm{E}$ & 5 & Rogers [21] \\
\hline $\begin{array}{l}\text { Montastraea annularis } \\
\text { (Ellis \& Solander, 1786) }\end{array}$ & $\begin{array}{l}800 \mathrm{mg} \mathrm{cm}^{-2} \mathrm{~d}^{-1} \text { (single } \\
\text { application) }\end{array}$ & Mortality & $\mathrm{M} / \mathrm{E}$ & 5 & Rogers [21] \\
\hline $\begin{array}{l}\text { Montastraea annuiaris } \\
\text { (Ellis \& Solander, 1786) }\end{array}$ & $430 \mathrm{mg} \mathrm{cm}^{-2} \mathrm{~d}^{-1}$ (sand + oil) & Mortality after 1 day & $\mathrm{L} / \mathrm{M}$ & 5 & $\begin{array}{c}\text { Bak \& Elgetshuizen } \\
{[63]}\end{array}$ \\
\hline $\begin{array}{l}\text { Montastraea annularis } \\
\text { (Ellis \& Solander, 1786) }\end{array}$ & $10 \mathrm{mg} \mathrm{cm}^{-2} \mathrm{~d}^{-1}$ (natural) & Reduced \%cover & M & 5 & $\begin{array}{c}\text { Torres \& Morelock } \\
{[87]}\end{array}$ \\
\hline $\begin{array}{l}\text { Montastraea annularis } \\
\text { (Ellis \& Solander, 1786) }\end{array}$ & $\begin{array}{l}19 \mathrm{mg} \mathrm{cm}^{-2} \mathrm{~d}^{-1} \text { (resuspended } \\
\text { carbonate mud) }\end{array}$ & Reduced growth rate & M & 5 & Dodge et al. [28] \\
\hline $\begin{array}{l}\text { Montastraea annuiaris } \\
\text { (Ellis \& Solander, 1786) }\end{array}$ & $\begin{array}{c}\text { Burial (10-12 cm of reef } \\
\text { sand) }\end{array}$ & $\begin{array}{c}40 \% \text { Tissue loss within } 24 \\
\text { h; } 90 \% \text { tissue loss within } \\
72 \mathrm{~h}\end{array}$ & M & 5 & Thompson [64] \\
\hline $\begin{array}{l}\text { Montasrraea annularts } \\
\text { (Ellis \& Solander, 1786) }\end{array}$ & - & $\begin{array}{l}\text { Produces little mucus; re- } \\
\text { moves silt by ciliary action }\end{array}$ & M & 5 & $\begin{array}{c}\text { Dumas \& Thomassin } \\
{[86]}\end{array}$ \\
\hline $\begin{array}{l}\text { Montastraea cavernosa } \\
\quad \text { (Linnaeus, 1767) }\end{array}$ & $<1390 \mathrm{mg} \mathrm{cm}^{-2} \mathrm{~d}^{-1}$ & Survival & M & 11 & Lasker [89] \\
\hline $\begin{array}{l}\text { Montastraea cavernosa } \\
\quad \text { (Linnaeus, 1767) }\end{array}$ & $150 \mathrm{mg} / \mathrm{m}^{2} / \mathrm{d}$ (natural) & Survival/dominance & M & 11 & Loya [74] \\
\hline $\begin{array}{l}\text { Montastraea cavernosa } \\
\quad \text { (Linnaeus, 1767) }\end{array}$ & $\begin{array}{l}\text { Burial }(10-12 \mathrm{~cm} \text { of reef } \\
\text { sand })\end{array}$ & $\begin{array}{l}30 \% \text { Tissue loss after } 72 \mathrm{~h}: \\
\text { remaining tissue in decay }\end{array}$ & M & 11 & Thompson [64] \\
\hline
\end{tabular}




\begin{tabular}{|c|c|c|c|c|c|}
\hline Coral species & $\begin{array}{l}\text { Sedimentation rate (test- } \\
\text { ed) }\end{array}$ & Response & Growth form & Calyx (mm) & References \\
\hline $\begin{array}{l}\text { Montipora aequitubercula- } \\
\text { ta (Bernard, 1897) }\end{array}$ & $200 \mathrm{mg} \mathrm{cm}^{-2} \mathrm{~d}^{-1}$ & $\begin{array}{c}\text { Bleaching after } 6 \text { days (but } \\
\text { no mortality) }\end{array}$ & $\mathrm{L}$ & 0.6 & Stafford-Smith [48] \\
\hline $\begin{array}{l}\text { Montipora aequitubercula- } \\
\text { ta (Bernard, 1897) }\end{array}$ & $\begin{array}{c}25 \mathrm{mg} \mathrm{cm}^{-2} \mathrm{~d}^{-1} \text { (up to } 16 \\
\text { weeks) }\end{array}$ & $\begin{array}{c}\text { Onset mortality after } 4 \\
\text { weeks, full mortality after } \\
12 \text { weeks }\end{array}$ & $\mathrm{F}$ & 0.6 & $\begin{array}{l}\text { Negri et al. [90] \& } \\
\text { Flores et al. [33] }\end{array}$ \\
\hline $\begin{array}{c}\text { Montipora capdata (Dana, } \\
1846 \text { ) }\end{array}$ & $\begin{array}{l}\text { Burial }\left(2.2-2.8 \mathrm{~g} / \mathrm{cm}^{2}\right. \\
\text { for } 45 \mathrm{~h})\end{array}$ & $\begin{array}{l}\text { sub lethal effects after } 30 \\
\mathrm{~h} \text {, little recovery after } 90 \mathrm{~h}\end{array}$ & B & 2 & Pinlaic [91] \\
\hline $\begin{array}{c}\text { Montipora foliosa (Pallas, } \\
1766 \text { ) }\end{array}$ & - & Active sediment rejecter & $\mathrm{L}$ & 0.7 & $\begin{array}{l}\text { Stafford-Smith \& } \\
\text { Ormond [75] }\end{array}$ \\
\hline $\begin{array}{l}\text { Montipora peltifortnis } \\
\text { (Bernard, 1897) }\end{array}$ & $\begin{array}{c}33-160 \mathrm{mg} / \mathrm{cm}^{2} \text { (silt) expo- } \\
\text { sure for } 36 \mathrm{~h}\end{array}$ & $\begin{array}{l}\text { Reduced photosynthesis } \\
\text { within } 12-60 \mathrm{~h}\end{array}$ & $\mathrm{~F}$ & 1 & Weber et al. [92] \\
\hline $\begin{array}{l}\text { Montipora peltifortnis } \\
\text { (Bernard, 1897) }\end{array}$ & $79-234 \mathrm{mg} / \mathrm{cm}^{2}$ (up to $36 \mathrm{~h}$ ) & $\begin{array}{c}\text { Significant decline in } \\
\text { photosynthesis (quantum } \\
\text { yield) }\end{array}$ & $\mathrm{M} / \mathrm{L}$ & 1 & $\begin{array}{c}\text { Philipp \& Fabricius } \\
\text { [93] }\end{array}$ \\
\hline Montipora spp. & $\left(0.9-1.3 \mathrm{mg} / \mathrm{m}^{2} /\right.$ day $)$ & $\begin{array}{l}\text { Described as 'sensitive' to } \\
\text { sedimentation }\end{array}$ & - & - & $\begin{array}{c}\text { McClanahan \& Obura } \\
\text { [83] }\end{array}$ \\
\hline $\begin{array}{l}\text { Montipora verrucosa (La- } \\
\text { marck, 1816) }\end{array}$ & $\begin{array}{c}30 \mathrm{mg} \mathrm{cm}^{-2} \mathrm{~d}^{-1} \text { (daily appli- } \\
\text { cations) }\end{array}$ & $\begin{array}{l}\text { Survived (10 days of appli- } \\
\text { cation) }\end{array}$ & M & 1.5 & Hodgson [94] \\
\hline $\begin{array}{l}\text { Mycetaphyllia aliciae } \\
\text { (Wells, 1973) }\end{array}$ & $430 \mathrm{mg} \mathrm{cm}^{-2} \mathrm{~d}^{-1}($ sand + oil $)$ & Mortality after 1 day & $\mathrm{L}$ & 14 & $\begin{array}{c}\text { Bak \& Eigershulzen } \\
{[63]}\end{array}$ \\
\hline $\begin{array}{c}\text { Oxypora giabra (Nemenzo, } \\
1959 \text { ) }\end{array}$ & $\begin{array}{c}30 \mathrm{mg} \mathrm{cm}^{-2} \mathrm{~d}^{-1} \text { (daily appli- } \\
\text { cations) }\end{array}$ & $\begin{array}{l}\text { Total mortality within } 10 \\
\text { days }\end{array}$ & $\mathrm{L} / \mathrm{E}$ & 5 & Hodgson [94] \\
\hline $\begin{array}{c}\text { Pectinia lactuca (Pallas, } \\
1766)\end{array}$ & - & Active sediment rejecter & $\mathrm{L}$ & 18 & $\begin{array}{l}\text { Stafford-Smith \& } \\
\text { Ormond [75] }\end{array}$ \\
\hline
\end{tabular}




\begin{tabular}{|c|c|c|c|c|c|}
\hline Coral species & $\begin{array}{l}\text { Sedimentation rate (test- } \\
\text { ed) }\end{array}$ & Response & Growth form & Calyx (mm) & References \\
\hline $\begin{array}{l}\text { Pectinia paeonia (Dana, } \\
\text { 1846) }\end{array}$ & - & Active sediment rejecter & L & 15 & $\begin{array}{l}\text { Stafford-Smith \& } \\
\text { Ormond [75] }\end{array}$ \\
\hline Pectinia sp. & - & Active sediment rejecter & - & - & $\begin{array}{l}\text { Stafford-Smith \& } \\
\text { Ormond [75] }\end{array}$ \\
\hline $\begin{array}{l}\text { Platygyra daertalea (Ellis \& } \\
\text { Solander, 1786) }\end{array}$ & $200 \mathrm{mg} \mathrm{cm}^{-2} \mathrm{~d}^{-1}$ ( 6 weeks) & $\begin{array}{l}\text { Minor tissue damage, } \\
\text { mucus production, no } \\
\text { bleaching }\end{array}$ & M & 5 & $\begin{array}{l}\text { Riegl [81] \& Riegl \& } \\
\text { Bloomer [82] }\end{array}$ \\
\hline $\begin{array}{l}\text { Platygyra sinensis (Milne } \\
\text { Edwards \& Haime, 1849) }\end{array}$ & Complete burial & $\begin{array}{l}\text { Bleaching and tissue dam- } \\
\text { age after } 48 \mathrm{~h}\end{array}$ & M & 4 & Wong [95] \\
\hline Platygyra spp. & $\left(4 \mathrm{mg} / \mathrm{m}^{2} /\right.$ day $)$ & $\begin{array}{c}\text { Tolerance to sedimenta- } \\
\text { tion described as 'inter- } \\
\text { mediate.' }\end{array}$ & - & - & $\begin{array}{c}\text { McClanahan \& Obura } \\
\text { [83] }\end{array}$ \\
\hline $\begin{array}{l}\text { Pteuractis granutosa } \\
\text { (Klunzinger, 1879) }\end{array}$ & $\begin{array}{l}\text { Continuously repeated burial } \\
\text { (sand) }\end{array}$ & $\begin{array}{l}\text { Survival (high endurance } \\
\text { with no apparent effect) }\end{array}$ & S & 185 & Schuhmacher [77] \\
\hline $\begin{array}{l}\text { Pteuractis granulosa } \\
\text { (Klunzinger, 1879) }\end{array}$ & $15 \mathrm{~mm}^{3} / \mathrm{cm}^{2} / \mathrm{d}$ & Maximum rate tolerated & S & 185 & Schuhmacher [77] \\
\hline $\begin{array}{l}\text { Pteuractis moluccensis } \\
\text { (Van der Horst. 1919) }\end{array}$ & - & $\begin{array}{l}\text { Adapted to withstand } \\
\text { considerable sedimenta- } \\
\text { tion rates }\end{array}$ & $S$ & 19 & Schuhmacher [77] \\
\hline $\begin{array}{l}\text { Pocillopora damicornis } \\
\quad \text { (Linnaeus, 1758) }\end{array}$ & $50-95 \%$ sediment cover & $\begin{array}{l}\text { Complete inhibition of } \\
\text { larval settlement }\end{array}$ & B & 1 & Hodgson [96] \\
\hline $\begin{array}{l}\text { Pocillopora damicornis } \\
\text { (Linnaeus, 1758) }\end{array}$ & $\begin{array}{l}67 \text { and } 186 \mathrm{mg} \mathrm{cm}^{-2} \mathrm{~d}^{-1} \text { (fine } \\
\text { silt; } 83 \text { days) }\end{array}$ & $\begin{array}{l}50-100 \% \text { Mortality of } \\
\text { transplanted (esp. small) }\end{array}$ & B & 1 & Sakai et al. [97] \\
\hline
\end{tabular}




\begin{tabular}{|c|c|c|c|c|c|}
\hline Coral species & $\begin{array}{l}\text { Sedimentation rate (test- } \\
\text { ed) }\end{array}$ & Response & Growth form & Calyx (mm) & References \\
\hline $\begin{array}{l}\text { Pocillopora darnicomis } \\
\text { (Linnaeus. 1758) }\end{array}$ & $\begin{array}{c}11-490 \mathrm{mg} \mathrm{cm}^{-2} \mathrm{~d}^{-1}(11 \\
\text { months })\end{array}$ & $\begin{array}{l}\text { The reduced growth rate } \\
\text { of transplanted }\end{array}$ & B & 1 & Piniak \& Brown [98] \\
\hline $\begin{array}{l}\text { Pocillopora meandrina } \\
\text { (Dana, 1846) }\end{array}$ & $\begin{array}{c}30 \mathrm{mg} \mathrm{cm}^{-2} \mathrm{~d}^{-1} \text { (daily appli- } \\
\text { cations) }\end{array}$ & Mortality within 10 days & B & 1 & Hodgson [94] \\
\hline Pocillopora sp. & $\begin{array}{l}\text { Increased sedimentation } \\
\text { (dredging) }\end{array}$ & Considerable mortality & - & - & Hudson et al. [99] \\
\hline Pocillopora app. & $\left(0.9-1.3 \mathrm{mg} / \mathrm{m}^{2} /\right.$ day $)$ & $\begin{array}{l}\text { Described as 'sensitive' to } \\
\text { sedimentation }\end{array}$ & - & - & $\begin{array}{c}\text { McClanahan \& Obura } \\
{[83]}\end{array}$ \\
\hline $\begin{array}{l}\text { Porites astreoides (La- } \\
\text { marck, 1816) }\end{array}$ & $\begin{array}{l}\text { Heavy sedimentation event } \\
\qquad(>1 \mathrm{~cm})\end{array}$ & $\begin{array}{l}\text { Mortality (inability to } \\
\text { reject sediment) }\end{array}$ & $\mathrm{L}$ & 1.5 & Bak [36] \\
\hline $\begin{array}{l}\text { Porites astreoides (La- } \\
\text { marck, 1816) }\end{array}$ & - & $\begin{array}{l}\text { Abundant in heavily sedi- } \\
\text { mented areas }\end{array}$ & M & 1.5 & Cortes \& Risk [100] \\
\hline $\begin{array}{l}\text { Porites astreoides (La- } \\
\text { marck, 1816) }\end{array}$ & $430 \mathrm{mg} \mathrm{cm}^{-2} \mathrm{~d}^{-1}$ (sand) & Mortality after 1 day & $\mathrm{M} / \mathrm{E}$ & 1.5 & $\begin{array}{l}\text { Bak \& Elgershuizen } \\
{[63]}\end{array}$ \\
\hline $\begin{array}{c}\text { Porites astreoides (La- } \\
\text { marck, 1816) }\end{array}$ & $10 \mathrm{mg} \mathrm{cm}^{-2} \mathrm{~d}^{-1}$ (natural) & No effect & $\mathrm{M} / \mathrm{E}$ & 1.5 & $\begin{array}{c}\text { Torres arid Morelock } \\
\text { [87] }\end{array}$ \\
\hline $\begin{array}{l}\text { Porites astreoides (La- } \\
\text { marck, 1816) }\end{array}$ & $\begin{array}{l}\text { Burial }(10-12 \mathrm{~cm} \text { of reef } \\
\text { sand })\end{array}$ & $\begin{array}{l}\text { Bleaching within } 24 \mathrm{~h} \\
70 \% \text { tissue loss after } 72 \mathrm{~h}\end{array}$ & $\mathrm{M} / \mathrm{E}$ & 1.5 & Thompson [64] \\
\hline $\begin{array}{l}\text { Porites lobata (Dana, } \\
\text { 1846) }\end{array}$ & $\begin{array}{c}30 \mathrm{mg} \mathrm{cm}^{-2} \mathrm{~d}^{-1} \text { (daily appli- } \\
\text { cations) }\end{array}$ & Mortality within 10 days & M & 1.5 & Hodgson [94] \\
\hline $\begin{array}{c}\text { Porites lobata (Dana, } \\
1846)\end{array}$ & $\begin{array}{c}\text { Burial }\left(1.5-1.6 \mathrm{~g} / \mathrm{cm}^{2} \text { for }\right. \\
45 \mathrm{~h})\end{array}$ & $\begin{array}{l}\text { Sub lethal effects after } 30 \\
\text { h, little recovery after } 90 \mathrm{~h}\end{array}$ & M & 1.5 & Piniak [91] \\
\hline
\end{tabular}




\begin{tabular}{|c|c|c|c|c|c|}
\hline Coral species & $\begin{array}{l}\text { Sedimentation rate (test- } \\
\text { ed) }\end{array}$ & Response & Growth form & Calyx (mm) & References \\
\hline $\begin{array}{c}\text { Porites lobata (Dana, } \\
\text { 1846) }\end{array}$ & $200 \mathrm{mg} \mathrm{cm}^{-2} \mathrm{~d}^{-1}$ & $\begin{array}{c}\text { Bleaching after } 6 \text { days (but } \\
\text { no mortality) }\end{array}$ & M & 1.5 & Stafford-Smith [48] \\
\hline $\begin{array}{l}\text { Porites lobata (Dana, } \\
\text { 1846) }\end{array}$ & Complete burial (48 h) & $\begin{array}{l}\text { Bleaching; full recovery } \\
\text { after sediment removal }\end{array}$ & M & 1.5 & Yeung [101] \\
\hline $\begin{array}{l}\text { Porites lutea Milne (Ed- } \\
\text { wards \&Haime, 1851) }\end{array}$ & $200 \mathrm{mg} \mathrm{cm}^{-2} \mathrm{~d}^{-1}$ & $\begin{array}{c}\text { Bleaching after } 6 \text { days (but } \\
\text { no mortality) }\end{array}$ & M & 1.5 & Stafford-Smith [48] \\
\hline $\begin{array}{l}\text { Porites lutea Milne (Ed- } \\
\text { wards \&Haime, 1851) }\end{array}$ & - & $\begin{array}{l}\text { Common on reefs affected } \\
\text { by sedimentation }\end{array}$ & M & 1.5 & $\begin{array}{c}\text { Brown and Howard } \\
{[84]}\end{array}$ \\
\hline $\begin{array}{l}\text { Porites lutea Milne (Ed- } \\
\text { wards \&Haime, 1851) }\end{array}$ & $\begin{array}{l}\text { Increased sedimentation } \\
\text { (dredging) }\end{array}$ & Survival & M & 1.5 & Hudson et al. [99] \\
\hline $\begin{array}{l}\text { Porites lutea Milne (Ed- } \\
\text { wards \&Haime, 1851) }\end{array}$ & $\begin{array}{l}\text { Up to } 14.6 \mathrm{mg} / \mathrm{m}^{2} / \mathrm{d} \text { (fine silt } \\
\text { due to dredging) }\end{array}$ & $\begin{array}{l}\text { No effect on growth rate } \\
\text { (in situ) }\end{array}$ & M & 1.5 & Chansang et al. [65] \\
\hline $\begin{array}{l}\text { Porites parties (Pallas, } \\
\text { 1766) }\end{array}$ & - & $\begin{array}{l}\text { Uses tentacles to remove } \\
\text { larger sediment particles }\end{array}$ & M & 2 & Meyer [102] \\
\hline $\begin{array}{l}\text { Porites porites (Pallas, } \\
\text { 1766) famia furcata }\end{array}$ & $\begin{array}{c}\text { Burial }(10-12 \mathrm{~cm} \text { of reef } \\
\text { sand })\end{array}$ & $\begin{array}{c}90 \% \text { bleaching within } 24 \\
\text { hrs.; } 70 \% \text { tissue loss after } \\
72 \text { hrs. }\end{array}$ & B & 2 & Thompson [64] \\
\hline Porite.s rus (Forskal, 1775) & $39.6 \mathrm{mg} \mathrm{cm}^{-2} \mathrm{~d}^{-1}$ (for 2 weeks) & Massive mortality (anoxia) & M & 0.5 & Fabricius et al. [22] \\
\hline Porites sp. & - & $\begin{array}{l}\text { Persists in areas of heavy } \\
\text { sedimentation }\end{array}$ & - & - & Fabricius [100] \\
\hline Porites sp. & Burial for $6 \mathrm{~h}$ & No effect & - & - & Wesseling et al. [71] \\
\hline Porites sp. & Burial for $20 \mathrm{~h}$ & $\begin{array}{l}\text { Discoloration \& bleaching } \\
\text { after } 3 \text { weeks }\end{array}$ & - & - & Wesseling et al. [71] \\
\hline Porites sp. & $39.6 \mathrm{mg} \mathrm{cm}^{-2} \mathrm{~d}^{-1}$ (for 2 weeks) & $\begin{array}{l}\text { Mucus production, survival } \\
\text { (most tolerant) }\end{array}$ & - & - & Fabricius et al. [72] \\
\hline
\end{tabular}




\begin{tabular}{|c|c|c|c|c|c|}
\hline Coral species & $\begin{array}{l}\text { Sedimentation rate (test- } \\
\text { ed) }\end{array}$ & Response & Growth form & Calyx (mm) & References \\
\hline Porites spp, & $\begin{array}{c}\text { (between } 1.3 \text { and } 4 \mathrm{mg} \mathrm{cm}^{-2} \\
\mathrm{~d}^{-1} \text { not quoted) }\end{array}$ & $\begin{array}{l}\text { Tolerance to sedimenta- } \\
\text { tion described as 'inter- } \\
\text { mediate.' }\end{array}$ & - & - & $\begin{array}{c}\text { McClanahan \& Obura } \\
\text { [83] }\end{array}$ \\
\hline $\begin{array}{l}\text { Sarcophyton glaucum } \\
\text { (Quay \& Gaimard, 1833) }\end{array}$ & $200 \mathrm{mg} \mathrm{cm}^{-2} \mathrm{~d}^{-1}$ & $\begin{array}{l}\text { Tissue damage and partial } \\
\text { mortality within } 6 \text { weeks }\end{array}$ & So & - & Riegl [81] \\
\hline $\begin{array}{l}\text { Scolymia cubensis (Milne } \\
\text { Edwards \& Haime, 1849) }\end{array}$ & Complete burial & $50 \%$ Survival after 7 days & S & 75 & Rice \& Hunter [37] \\
\hline $\begin{array}{l}\text { Scolymia cubensis (Milne } \\
\text { Edwards \& Haime, 1849) }\end{array}$ & $\begin{array}{l}3 \mathrm{~g} \text { of } 3 \text { grain-sizes: } 62 \mu \mathrm{m}, \\
250 \mu \mathrm{m}, 2 \mathrm{~mm}(24 \mathrm{~h})\end{array}$ & $\begin{array}{l}\text { Sediment-shedding } \\
\text { efficiency related to calicle } \\
\text { angle }\end{array}$ & $S$ & 75 & Logan [106] \\
\hline $\begin{array}{l}\text { Siderastrea radians (Pallas. } \\
1766 \text { ) }\end{array}$ & Complete burial & $\begin{array}{c}50 \% \text { Survival after } 13,6 \\
\text { days }\end{array}$ & $\mathrm{M} / \mathrm{E}$ & 5 & Rice \& Hunter [37] \\
\hline $\begin{array}{c}\text { Siderastrea radians (Pallas, } \\
1766 \text { ) }\end{array}$ & Total burial & $\begin{array}{l}\text { Survival for more than } \\
73 \mathrm{~h}\end{array}$ & $\mathrm{M} / \mathrm{E}$ & 5 & Mayer [107] \\
\hline $\begin{array}{c}\text { Siderastrea radians (Pallas, } \\
1766 \text { ) }\end{array}$ & Burial (chronic) & $\begin{array}{l}\text { Reduced growth and some } \\
\text { mortality }\end{array}$ & $\mathrm{M} / \mathrm{E}$ & 5 & Lirman et al. [108] \\
\hline $\begin{array}{l}\text { Siderastrea siderea (Ellis \& } \\
\text { Solander, 1786) }\end{array}$ & $10 \mathrm{mg} \mathrm{cm}^{-2} \mathrm{~d}^{-1}$ (natural) & No effect & M & 3 & $\begin{array}{c}\text { Torres \& Morelock } \\
{[87]}\end{array}$ \\
\hline $\begin{array}{l}\text { Siderastrea siderea (Ellis \& } \\
\text { Solander, 1786) }\end{array}$ & $0.3-64 \mathrm{mg} \mathrm{cm}^{-2} \mathrm{~d}^{-1}$ & Partial mortality & M & 3 & $\begin{array}{c}\text { Nugues \& Roberts } \\
{[109]}\end{array}$ \\
\hline $\begin{array}{c}\text { Siderastrea siderea (Ellis \& } \\
\text { solander. 1786) }\end{array}$ & $\begin{array}{l}\text { Burial }(10-12 \mathrm{~cm} \text { of reef } \\
\text { sand })\end{array}$ & $\begin{array}{l}50 \% \text { Bleaching and sub } \\
\text { lethal stress within } 24 \mathrm{hr} \text {. }\end{array}$ & M & 3 & Thompson [64] \\
\hline $\begin{array}{c}\text { Sinularia tiara (Pratt, } \\
\text { 1903) }\end{array}$ & $200 \mathrm{mg} \mathrm{cm}^{-2} \mathrm{~d}^{-1}$ ( 6 weeks) & $\begin{array}{c}\text { Minor tissue damage and } \\
\text { bleaching }\end{array}$ & So & - & $\begin{array}{l}\text { Riegl [81] \& Riegl \& } \\
\text { Bloomer [82] }\end{array}$ \\
\hline
\end{tabular}




\begin{tabular}{|c|c|c|c|c|c|}
\hline Coral species & $\begin{array}{l}\text { Sedimentation rate (test- } \\
\text { ed) }\end{array}$ & Response & Growth form & Calyx (mm) & References \\
\hline $\begin{array}{l}\text { Sinularia leptoclados (Eh- } \\
\text { renberg,1834) }\end{array}$ & $200 \mathrm{mg} \mathrm{cm}^{-2} \mathrm{~d}^{-1}$ (6 weeks) & $\begin{array}{l}\text { Minor tissue damage and } \\
\text { bleaching }\end{array}$ & So & - & $\begin{array}{l}\text { Riegl [81] \& Riegl \& } \\
\text { Bloomer [82] }\end{array}$ \\
\hline $\begin{array}{c}\text { Solenastrea hyades (Dana, } \\
1846 \text { ) }\end{array}$ & Complete burial & $\begin{array}{c}50 \% \text { Survival after }>15 \\
\text { days }\end{array}$ & M & 5 & Rice \& Hunter [37] \\
\hline $\begin{array}{l}\text { Stephanocoenia iniersepta } \\
\text { (Lamarck, 1816) }\end{array}$ & Complete burial & $\begin{array}{l}50 \% \text { Survival after } 16.2 \\
\text { days }\end{array}$ & M & 3 & Rice \& Hunter [37] \\
\hline $\begin{array}{l}\text { Trachyphyllia geoffroyi } \\
\text { (Audouin, 1826) }\end{array}$ & - & $\begin{array}{l}\text { Actively dig through over- } \\
\text { lying sediment }\end{array}$ & $S$ & 45 & $\begin{array}{l}\text { Stafford-Smith \& } \\
\text { Ormond [75] }\end{array}$ \\
\hline Turbinaria mesenterina & $110 \mathrm{mg} / \mathrm{cm}^{2}$ (5 weeks) & $\begin{array}{l}\text { No significant sub lethal } \\
\text { physiological effects }\end{array}$ & $\mathrm{L}$ & 1.5 & $\begin{array}{c}\text { Sofonia and Anthony } \\
{[110]}\end{array}$ \\
\hline Turbinarta (several spp.) & - & Active sediment rejecter & - & - & $\begin{array}{l}\text { Stafford-Smith \& } \\
\text { Ormond [75] }\end{array}$ \\
\hline
\end{tabular}

However, the background concentration derived by Caroline [60] found to be suitable for the earlier studies of at Palk bay \& Lakshadweep recorded minimum of 1 to $2.69 \mathrm{mg} \mathrm{cm}^{-2} \mathrm{~d}^{-1}$ during the fair-weather periods. Investigations on effects of sediment stress in 89 coral species provided a generic understanding of tolerance levels, response mechanisms, adaptations and threshold levels of corals to the effects of natural and anthropogenic sediment disturbances Paul et al. [19]. The algal symbionts of coral polyps undergo stress from high suspended sediment concentrations and the subsequent effects on light attenuation. The bare minimum light requirements of corals reef ranges from $<1 \%$ to as much as $60 \%$ of surface irradiance Paul et al. [19]. However, the chronic levels of suspended sediment load range between $<10 \mathrm{mg} \mathrm{L}^{-1}$ in pristine offshore reef areas to $>100 \mathrm{mg} \mathrm{L}^{-1}$ in marginal near shore reefs Paul et al. [19]. But the tolerance level of exogenous sedimentation rates for different corals species ranged between $<10 \mathrm{mg} \mathrm{cm}^{-2} \mathrm{~d}^{-1}$ to $>400 \mathrm{mg} \mathrm{cm}^{-2} \mathrm{~d}^{-1}$. The exposure duration of high sedimentation rates varied from $<24 \mathrm{~h}$ for sensitive species to a few weeks ( $>4$ weeks of high sedimentation or $>14$ days complete burial) for very tolerant species. This quantification of sensitivity between different coral species was accounted by the growth form of coral colonies and the size of the coral polyp or calyx. These observations were derived from the 77 published studies on the effects of turbidity and sedimentation on 89 coral species Paul et al. [19] and presented in tabular from [Table 4 \& 5]. Most of the case studies depict discrete turbidity or sedimentation events which produce stress on coral reefs [21]. In India, existing knowledge indicates that inshore corals in certain regions like Gulf of Kutch region may have adapted to high turbidity regimes. According to Anthony \& Larcombe [61], coral resilience to turbidity might be of rapid replenishment of energy reserves between periods of sublethal turbidity events, interchanging between phototrophic and heterotrophic dependence, rapid rates of photo-acclimation and energy conservation through reduced respiratory and excretory losses. The current record of the occurrence of symbiodinium spp. at Gulf of Kutch express the physiological resilience is also documented Koushik et al. [62]. These stress response of Indian coral reefs necessitates a comprehensive scientific approach towards solving the siltation problem through technological intervention. The precursor for any technological intervention requisite understanding of the different process governing the sedimentation shall be carried out by numerical modelling. The modeling effort has been warranted only to reef environment where the exogenous sedimentation from the natural environment exceeds endogenous sedimentation from the reef itself. 


\section{Technological Interventions}

In general, standard engineering modeling tool shall be used to quantitatively simulate sediment transport and deposition with in a system [109]. Hence, conceptual site specific modelling for various sites in India needs to be developed to understand dynamic sediment transport along coral reef systems. As coral reefs have a unique environment, comprehensive understandings of the physical, chemical and biological processes influencing the fate and transport of sediments laden with contaminants of potential concern from sources to exposure media (ie. coral reefs). Hence, a conceptual numerical model shall be developed comprising vital components like hydrodynamic and sediment transport. These components were adequately described by various processes like terrigenous matter inflow through rivers, tidal forcing, meteorological conditions, sediment size gradation, sediment bed properties, advection, dispersion, aggregation, settling, consolidation, erosion transport in suspension, water quality and particle-to-particle interactions and anthropogenic activities of current as well as historical condition. Accounting all these components a numerical model using Mike21, Delf 3D shall be set up to mimic natural conditions. The dynamics of these components shall be simulated using the numerical modelling and calibrated against observed values. Latter validated against the time series field data set collected in a specific manner as per the model requirement. The model output will be of the scenarios of the current, historical and future condition of sedimentation in a reef environment under consideration. The output of the modeling exercise will reveal the impact of siltation due to anthropogenic input (industrial discharge) or other factors like river runoff, bank erosion, etc. If the anthropogenic input predominates the other means of sedimentation, government departments like state pollution control boards and central pollution control boards shall be informed to curb the input through legislative means. However, the exogenous sedimentation rate found to be higher and segregate possible input areas of sediment load shall be mapped within the model domain, and suitable technological intervention may be suggested.

Sediment movement may be arrested by established technologies by providing geotube dykes along the eroding banks to prevent leach outs. As this technology found to be eco friendly and standardized by NIOT for coastal erosion prevention [110]. The model scenario of future condition after technological intervention also been simulated and try to achieve the sedimentation levels of $>100 \mathrm{mg} \mathrm{L}^{-1}$ or $>400 \mathrm{mg} \mathrm{cm}^{-2} \mathrm{~d}^{-1}$ as reported in the literature. Further studies are required to validate the maximum tolerable level of sedimentation level on coral reefs of Indian coast. The floating macro algal mass settlement on coral reefs of [111] was also reported during January to March. It may be prevented by floating barrier capable of filtering macro algae and not entangle fish as well as turtles. The further large scale is floating algae; garbage netting and collection by [112] may be adopted. This innovative boat capable of collecting floating macro algae materials with its foldable arms covering a width of $4 \mathrm{~m}$ span and collection rate of
$98 \mathrm{~m}^{3}$ day $^{-1}$ during operation. Further, coral reef rehabilitation shall be achieved through Biorock technology which uses mineral accretion process developed by Thomas Goreau, a marine biologist and Wolf Hilbertz, an engineer and architect [113-117] through electro deposition of calcium from sea water enhances the coral growth and the technology found to be viable at certain pockets of world reefs $[48,118,119]$.

\section{Conclusion}

Conservation efforts also been achieved through judicial utilization of current technological means.

\section{References}

1. Steven D, Quistada, Aleksandr Stotlanda, Katie LB, Cameron A, et al. (2014) Evolution of TNF-induced apoptosis reveals 550 of functional conservation. Proceedings of the National Academy of Sciences (PNAS) 111(26): 9567-9572

2. Barnosky AD, Matzke N, Tomiya S, Wogan GOU, Swartz B, et al. (2011) Has the Earth's sixth mass extinction already arrived? Nature 471(7336): 51-57.

3. Jablonski D (1994) Extinctions in the fossil record. Philosophical Trans actions of the Royal Society B 344(1307): 11-17.

4. Raup DM Sepkoski JJ (1982) Mass extinctions in the marine fossil record. Science 215(4539): 1501-1503.

5. Spalding MD, Grenfel AM (1977) New estimates of global and regional coral reef areas. Coral Reefs 16(4): 225-230.

6. Spalding M, Ravilious C, Green E (2001) World Atlas of Coral reefs. In: Spalding M, Ravilious C, Green E (Eds.), University of California Press and UNEP/WCMC, Berkeley, California, USA.

7. Chiras DD (1994) Environmental Science: Action for a sustainable future. In: Chiras DD (Ed.), Environmental Science: Action for a sustainable future. (4th edn), Redwood City: Benjamin/Cummings, USA, pp. 2.

8. Bruno JF, Selig ER (2007) Regional Decline of coral cover in the IndoPacific: Extent, Sub comparisons. PLoS One 2(8): e711.

9. Schutte VGW, Selig ER, Bruno JF (2010) Regional spatio-temporal trends in Caribbean coral reef benthic communities. Marine Ecology Progress Series 402: 115-122.

10. Burke L, Reytar K, Spalding M, Perry A (2011) Reefs at Risk Revisited. World Resources Institute, Washington DC, USA, pp. 1-130.

11. Amita Saxena (2015) Coral Reefs and Their Conservation - A Review. Biological and Chemical Research. pp. 187-206.

12. Saroj J, Gautam RK, Joshi A, Panja Tehseen (2016) Review of coral reefs of India: distribution, status, research and management. International Journal of Science Environment and Technology 5(5): 3088-3098.

13. Ginsburg RN, Glynn PW (1993) Summary of the colloquium and forum on the global aspects of coral reefs: health, hazards, and history. In: Ginsburg RNC (Ed.), Proceedings of the Colloquium on Global Aspects of Coral Reefs: Health, Hazards, and History. University of Miami, Rosenstiel School of Marine and Atmospheric Science, Florida.

14. Brown BE (1997) Disturbances to reefs in recent times. In: Birkeland C (Ed.), Life and death of coral reefs. Chapman and Hall, UK, pp. 354-385.

15. Richmond RH (1993) Coral reefs-present problems and future concerns resulting from anthropogenic disturbance. American Zoologist 33(6): 524-536

16. Dudley WC (2003) Coral reef sedimentology. Mare 461-Spring, pp. 1-23.

17. Pillai CSG (1986) Status of coral reefs in Lakshadweep. Marine Fisheries Information Service, Technical and Extension Series 68: 38-41. 
18. James PSBR, Gopinathan Pillai CS, Thomas PA, James DB, Said Koya, et a (1989) Environmental damages and its consequences. Bull Cent Mar fish Res Institute 43: 212-227.

19. Paul LA, Erftemeijer, Bernhard Riegl, Hoeksema BW, Peter A (2012) Environmental impacts of dredging and other sediment disturbances on corals: A review. Mar Pollut Bull 64(9): 1737-1765.

20. Suresh VR (1991) Studies on the coral reefs of Lakshadweep. Thesis Cochin University of Science and Technology India pp. 1-205.

21. Rogers CS (1990) Responses of coral reefs and reef organisms to sedimentation. Mar Ecol Prog Ser 62: 185-202.

22. Kumaraguru AK, Jayakumar, Kannan, Wilson Jerald, Muthiah Ramakritinan (2005) Impact of the tsunami of 26 December 2004 on the coral reef environment of Gulf of Mannar and Palk Bay in the southeast coast of India. Curr Sci 10 (2): 1729-1741.

23. Rogers CS (1979) The effect of shading on coral reef structure and function. Journal of Experimental Marine Biology and Ecology 41(3): 269-288.

24. Thornpson JH (1980b) Responses of selected scleractinian corals to drilling fluids used in the marine environment. PhD dissertation, Texas A \& M University, College Station.

25. Nieuwaal M (2001) Requirements for sediment plumes caused by dredging. MSc. Thesis, Delft University of Technology pp 89, Germany.

26. Kendall JJ, Powell EN, Connor SJ, Bright TJ (1983) The effects of drilling fluids (muds) and turbidity on the growth and metabolic state of the coral Acropora cervicornis, with comments on methods of normalization for coral data. Bulletin of Marine Science 33(2): 336-352.

27. Kendall JJ, Powell EN, Connor SJ, Bright TJ, Zastrow CE (1985) Effects of turbidity on calcification rate, protein concentrations and the free amino acid pool of the coral acropora cervicornis. Marine Biology 87(1): 33-46.

28. Thompson H, Bright TJ (1980) Effects of an offshore drilling fluid on selected corals. In: Proc. Research on Environmental fate and Effects of Drilling Fluids and Cuttings, Lake Buena Vista, Fla 2 1044-1076.

29. Gilmour J (1999) Experimental investigation into the effects of suspended sediment on fertilisation, larval survival and settlement in a scleractinian coral. Marine Biology 135(3): 451-456.

30. Anthony KRN (1999a) Coral suspension feeding on fine particulate matter. Journal of Experimental Marine Biology and Ecology 232: 85106.

31. Anthony KRN (2000) Enhanced particle-feeding of corals on turbid reefs (Great Barrier Reef, Australia). Coral Reefs 19(1): 59-67.

32. Negri AP, Flores F, Hoogen boom M, Abrego, Freckelton M, Cooper T (2009) Effects of dredging on shallow corals: experimental sediment exposure. Report to Woodside Energy: Browse Joint Venture Partners. Australian Institute of Marine Science, Townsville.

33. Flores FT, Cooper D, Abdo N, Webster Negri A (2011) A laboratorybased approach to understanding coral thresholds for dredging impact assessment. In: Paper presented at the 48th AMSA Conference, Fremantle, Book of Abstracts pp. 59.

34. Anthony KRN, Connolly SR, Hoegh-Guldberg (2007) Bleaching, energetics and coral mortality risk: effects of temperature, light, and sediment regime. Limnology and Oceanography 52(2): 716-726.

35. Fabricius KE, Wolanski E (2000) Rapid smothering of coral reef organisms by muddy marine snow. Estuarine and Coastal Shelf Science 50(1): 115-120.

36. Bak RPM (1978) Lethal and sublethal effects of dredging on reef corals. Marine Pollution Bulletin 9(1): 14-16.

37. Rice SA, Hunter CL (1992) Effects of suspended sediment and burial on scleractinian corals from West Central Florida patch reefs. Bulletin of Marine Science 51(3): 429-442.
38. Telesnicki GJ, Goldberg WM (1995) Effects of turbidity on the photosynthesis and respiration of two South Florida reef coral species. Bulletin Marine Science 57(2): 527-539.

39. Riegl B, Branch GM (1995) Effects of sediment on the energy budgets of four scleractinian (Bourne 1900) and five alcyonacean (Lamouroux 1816) corals. Journal of Experimental Marine Biology and Ecology 186(2): 259-275.

40. Dikou A, Van Woesik R (2006a) Partial colony mortality reflects coral community dynamics: a fringing reef study near a small river in Okinawa, japan. Marine Pollution Bulletin 52(3): 269-280.

41. Dikou A, Van Woesik R (2006b) Survival under chronic stress from sediment load: spatial patterns of hard coral communities in the southern islands of Singapore. Marine Pollution Bulletin 52(11): 13401354 .

42. Larcombe P, Costen A, Woolfe KJ (2001) The hydrodynamic and sedimentary setting of nearshore coral reefs, central Great Barrier Reef shelf, Australia: Paluma Shoals, a case study. Sedimentology 48(4): 811835.

43. Anthony KRN, Fabricius FE (2000) Shifting roles of heterotrophy and autotrophy in coral energetics under varying turbidity. Journal of Experimental Marine Biology and Ecology 252(2): 221-253.

44. Anthony KRN (1999b) A tank system for studying benthic aquatic organisms at predictable levels of turbidity and sedimentation: case study examining coral growth. Limnology and Oceanography 44(6): 1415-1422.

45. Fabricius KE, Dommisse M (2000) Depletion of suspended particulate matter over coastal reef communities dominated by zooxanthellate soft corals. Marine Ecology Progress Series 196: 157-167.

46. Froelich SS, Johnson V, Hoehn T (1981) The physiological effects of oildrilling muds on the caribbean coral montastrea annularis. Proceedings Fourth International Coral Reef Symposium 1: 163-168.

47. Dallmeyer DG, Porter JW, Smith GJ (1982) Effects of particulate peat on the behaviour and physiology of the Jamaican reef-building coral Montastrea annularis. Marine Biology 68(3): 229-233.

48. Smith SMG (1993) Sediment-rejection efficiency of 22 species of Australian scleractinian corals. Marine Biology 115(2): 229-243.

49. Piniak GA, Storlazzi CD (2008) Diurnal variability in turbidity and coral fluorescence on a fringing reef flat: southern molokai, hawaii. Estuarine, Coastal and Shelf Science 77(1): 56-64.

50. Anthony KRN, Guldberg H (2003) Kinetics of photoacclimation in corals. Oecologia 134(1): 23-31.

51. Te FT (1998) Turbidity and its effect on corals: a model using the extinction coefficient $(\mathrm{k})$ of photosynthetic active radiation (PAR). In: Proceedings Eighth International Coral Reef Symposium, Panama, pp. 1899-1904.

52. Erftemeijer PLA, Hagedorn M, Laterveer M, Craggs J, Guest JR (2012) Effect of suspended sediment on fertilization success in the scleractinian coral pectinia actuca. Journal of the Marine Biological Association of the United Kingdom 92(4): 741-745.

53. Brown BE, Tissier MDA, Scoffin TP, Tudhope AW (1990) Evaluation of the environmental impact of dredging on intertidal coral reefs at Ko phuket, thailand, using ecological and physiological parameters. Marine Ecology Progress Series 65: 273-281.

54. Tomascik T, Sander R (1987) Effects of eutrophication on reef-building corals. III. Reproduction of the reef-building coral porites porites. Marine Biology 94(1): 77-94.

55. Barnes DJ, Lough JM (1992) Systematic variations in the depth of skeleton occupied by coral tissue in massive colonies of porites from the great barrier reef. Journal of Experimental Marine Biology and Ecology 159(1): 113-128. 
56. Lough JM, Barnes DJ (1992) Comparisons of skeletal density variations in porites from the central great barrier reef. Journal of Experimental Marine Biology and Ecology 155(1): 1-25.

57. Lough JM, Barnes DJ (2000) Environmental controls on growth of the massive coral porites. Journal of Experimental Marine Biology and Ecology 245(2): 225-243.

58. Rice SA (1984) Effects of suspended sediment and burial upon survival and growth of Eastern Gulf of Mexico Corals. Camp Dresser \& McKee, Inc. Mote Marine Laboratory Technical Report No. 87 pp. 58.

59. Stoddart A, Stoddart SE. Eds. (2005) Corals of the Dampier Harbour: their survival and reproduction during the dredging programs of 2004 M Science Pty Ltd, University of Western Australia, Perth, Western Australia.

60. Caroline SR (1990) Responses of coral reefs and reef organisms to sedimentation. Mar Ecol Prog Ser 62: 185-202.

61. Anthony KRN, Larcombe P (2000) Coral reefs in turbid waters: sedimentinduced stresses in corals and likely mechanisms of adaptation. In Proceedings Ninth International Coral Reef Symposium, Bali, Indonesia, pp. 6.

62. Koushik S, Dholkia D, Bhadalkar L (2017) Genetic diversity of symbiodinium sp. to understand coral reef resilience in Gulf of kachchh. National Workshop on Coral Reef Ecosystem of West Coast of India: current status and Way Forward, at Jamnagar, Gujarat.

63. Bak RPM, Elgershuizen JHBW (1976) Patterns of oil sediment rejection in corals. Marine Biology 37(2): 715-730.

64. Thompson JHR (1980a) Effects of drilling mud on seven species of reefbuilding corals as measured in field and laboratory, In: Geyer, R.A. (Ed.), Marine Environmental Pollution pp. 433-454.

65. Chansang H, Phongsuwan N, Boonyanate P (1992) Growth of corals under effect of sedimentation along the northwest coast of Phuket Island, Thailand. In: Proceedings Seventh International Coral Reef Symposium, Guam, pp 241-248.

66. Simpson CJ (1988) Ecology of scleractinian corals in the dampier archipelago, western australia. Environmental Protection Authority, Perth, Technical Series 23: 215-227.

67. Babcock R, Davies P (1991) Effects of sedimentation on settlement of acropora millepora. Coral Reefs 9(4): 205-208.

68. Kojis BL, Quinn NJ (1984) Seasonal and depth variation in fecundity of acropora palijera at two reefs in papua new guinea. Coral Reefs 3(3): 165-172.

69. Salarn AH, Porter JW (1988) Physiological effects of sediment rejection on photosynthesis and respiration in three caribbean reef corals. Proceedings Sixth International Coral Reef Symposium Australia 2: 285292.

70. Rogers CS (1977) The response of a coral reef to sedimentation. PhD Thesis, University of Florida, Gainesville, pp.195.

71. Wesseling I, Uychiaoco AJ, Alifio PM, Aurin T, Vermaat JE (1999) Damage and recovery of four philippine corals from short-term burial. Marine Ecology Progress Series 176: 11-15.

72. Fabricius KE, Golbuu Y, Victor S (2007) Selective mortality in coastal reef organisms from an acute sedimentation event. Coral Reefs 26(1): 69-69.

73. Vanthof T (1983) The influence of dredging on a coral reef in bonaire, netherlands antilles. Paper presented at AIMLC Meeting, USA.

74. Loya Y (1976) Recolonization of Red Sea corals affected by natural catastrophes and man-made perturbations. Ecology 57(2): 278-289.

75. Smith SMG, Ormond RFG (1992) Sediment-rejection mechanisms of 42 species of Australian scleractinian corals. Australian Journal of Marine and Freshwater Research 43(4): 683-705.
76. Peters EC, Pilson MEQ (1985) A comparative study of the effects of sedimentation on symbiotic and asymbiotic colonies of the coral astrangia danae milne edwards and hime 1849. Journal of Experimental Marine Biology and Ecology 92(2-3): 215-230.

77. Schuhmacher H (1977) Ability of fungiid corals to overcome sedimentation. In: Proceedings Third International Coral Reef Symposium, Miami 1: 503-510.

78. Todd PA, Ladle RJ, Lewin Koh NJI, Chou LM (2004a) Genotype environment interactions in transplanted clones of the massive corals favia speciosa and diploastrea heliopora. Marine Ecology Progress Series 271: 167-182.

79. Rogers CS (1983) Sublethal and lethal effects of sediments applied to common caribbean reef corals in the field. Marine Pollution Bulletin 14(10): 378-382.

80. Dodge RE, Vaisnys JR (1977) Coral populations and growth patterns: responses to sedimentation and turbidity associated with dredging. Journal of Marine Research 35: 715-730.

81. Riegl B (1995) Effects of sand deposition on scleractinian and alcyonacean corals. Marine Biology 121(3): 517-526.

82. Riegl B, Bloomer JP (1995) Tissue damage in hard and soft corals due to experimental exposure to sedimentation. In: Proceedings tst European Regional Meeting ISKS, Vienna. Beitrage zur Palaeontologie Von Oesterreich 20: 51-63.

83. Clanahan T, Obura D (1997) Sediment effects on shallow coral reef communities in kenya. Journal of Experimental Marine Biology and Ecology 209(1-2): 103-122.

84. Brown BE, Howard LS (1985) Assessing the effects of stress on reef corals. Advances in Marine Biology 22: 1-63.

85. Smith MG (1992) Mortality of the hard coral leptoria phrygia under persistent sediment influx. In: Proceedings Seventh International Coral Reef Symposium, Guam 1: 289-299.

86. Dumas R, Thomassin BA (1977) Protein fractions in coral and zoantharian mucus: possible evolution in coral reef environments. In: Proceedings Third International Coral Reef Symposium 1: 517-524.

87. Torres J, Morelock J (2002) Effect of terrigenous sediment influx on coral cover and linear extension rates of three caribbean massive coral species. Caribbean Journal of Science 38: 222-229.

88. Dodge RE, Aller RC, Thompson J (1974) Coral growth related to resuspension of bottom sediments. Nature 247: 574-577.

89. Lasker HR (1980) Sediment rejection by reef corals: the roles of behavior and morphology in montastraea cavemosa (Linnaeus). Journal of Experimental Marine Biology and Ecology 47(1): 77-87.

90. Negri AP, Flores F, Hoogenboom M, Abrego, Freckelton M, et al. (2009) Effects of dredging on shallow corals: experimental sediment exposure. Report to Woodside Energy: Browse Joint Venture Partners. Australian Institute of Marine Science, Australia.

91. Piniak GA (2007) Effects of two sediment types on the fluorescence yield of two hawaiian scleractinian corals. Marine Environmental Research 64(4): 456-468.

92. Weber M, Lott C, Fabricius KE (2006) Sedimentation stress in a scleractinian coral exposed to terrestrial and marine sediments with contrasting physical, organic and geochemical properties. Journal of Experimental Marine Biology and Ecology 336(1): 18-32.

93. Philipp E, Fabricius K (2003) Photophysiological stress in scleractinian corals in response to short-term sedimentation. Journal of Experimental Marine Biology and Ecology 287(1): 57-78.

94. Hodgson G (1990a) Tetracycline reduces sedimentation damage to corals. Marine Biology 104(3): 493-496. 
95. Wong CC (2001) The responses of two scleractinian corals, platygyra sinensis and goniopora columna, to sedimentation and burial. MPhil. Thesis, The Chinese University of Hong Kong, Hong Kong.

96. Hodgson G (1990b) Sediment and the settlement of larvae of the reef coral pocillopora damicomis. Coral Reefs 9(1): 41-43.

97. Sakai K, Nishihira M, Kakinuma Y, Song JI (1989) A short-term field experiment on the effect of siltation on survival and growth of transplanted pocillopora damicomis branchlets. Galaxea 8: 143-156.

98. Piniak GA, Brown EK (2008) Growth and mortality of coral transplants (pocillopora damicornis) along a range of sediment influence in Maui, Hawaii. Pacific Science 62: 39-55.

99. Hudson JH, Shinn EA, Robbin DM (1982) Effects of offshore oil drilling on Philippine reef corals. Bulletin of Marine Science 32(4): 890-908.

100. Cortes J, Risk MJ (1985) A reef under siltation stress: cahuita, costa rica. Bulletin of Marine Science 36(2): 339-356.

101. Yeung KP (2000) Changes in zooxanthellae density and chlorophyll-a concentration in corals Porites lobata after short-term sediment burial. Directed Research Report, Environmental Science Programme, The Chinese University of Hong Kong, Hong Kong.

102. Meyer FO (1989) Corals under attack: can they fight back? In: Paper presented at the 1989 NAUIIQ, Reprint published in Conference Proceedings. DGS Field Guide Series (Red Sea Coastal Geology) pp. 2026.

103. Fabricius KE (2005) Effects of terrestrial runoff on the ecology of corals and coral reefs: review and synthesis. Marine Pollution Bulletin 50(2): 125-146

104. Logan A (1988) Sediment-shifting capability in the recent solitary coral Scolymia cubensis (Milne-Edwards and Haime) from Bermuda. Bulletin of Marine Science 43(2): 241-248.

105. Mayer AG (1918) Ecology of the murray island coral reef. Publications Carnegie Institute 183: 1-24.

106. Lirman D, Orlando B, Macia S, Manzello O, Kaufman L, et al. (2003) Coral communities of biscayne bay, florida and adjacent offshore areas: diversity, abundance, distribution, and environmental correlates. Aquatic Conservation: Marine and Freshwater Ecosystems 13(2): 121135.

107. Nugues MM, Roberts CM (2003) Partial mortality in massive reef corals as an indicator of sediment stress on coral reefs. Marine Pollution Bulletin 46(3): 314-323.
108. Sofonia JJ, Anthony KRN (2008) High-sediment tolerance in the reef coral turbinaria mesenterina from the inner Great barrier reef lagoon (Australia). Estuarine, Coastal and Shelf Science 78(4): 748-752.

109. Parmeshwar LS, Su HS, James SC, Shaller PJ, Doroudian M, et al. (2014) Conceptual site model for newark bay-hydrodynamics and sediment transport. J Mar Sci Eng 2: 123-139.

110. Kiran AS, JebaKumar PP, Ravichandran V, Abhishek T (2016) Detached segmented submerged breakwater made of geosynthetic tubes for kadalur periyakuppam coast, tamilnadu: a sustainable shoreline management solution. International Journal of Earth Sciences and Engineering 9(6): 2688-2694.

111. Sadhukhan K, Dholkia D, Bhadalkar AL (2017) Genetic diversity of symbiodinium spp to understand coral reef resilience in Gulf of Kachchh. National Workshop on Coral Reef Ecosystem of West Coast of India: Current Status and Way Forward, at Jamnagar, Gujarat, India.

112. http://www.theplayatimes.com/2017/05/03/the-sargaboat/

113. Hilbertz WH (1979) Electrodeposition of Minerals in Sea Water: Experiments and Applications. IEEE Journal on Oceanic Engineering 4(3): 94- 113

114. Hilbertz WH (1991) Solar-generated construction material from sea water to mitigate global warming. Building Research and Information 19: 242-255.

115. Bachtiar R (2003) Pengamatan Pembentukan Terumbu Buatan dengan Metode Mineral Accretion di Desa Pemuteran, Bali Barat. Skripsi Sarjana, Jurusan Ilmu dan Teknologi Kelautan FPIK, Institut Pertanian Bogor, Indonesia.

116. Hoegh-Guldberg $O$ (2015) Reviving the Ocean Economy: the case for action-2015. WWF International, Gland, Switzerland, pp.1-60.

117. Saroj J, Gautam RK, Joshi A, Panja Tehseen (2016) Review of coral reefs of India: distribution, status, research and management. International Journal of Science Environment and Technology 5(5): 3088-3098.

118. Jerald Wilson J, Marirnuthu N, Kumaraguru AK (2005) Sedimentation of silt in the coral reef environment of Palk Bay, India. J Mar bio Ass India 47(1): 83-87.

119. Quistada SD, Stotlanda A, Barotta KL, Smurthwaitea CA, Hiltona BJ, et al. (2014) Evolution of TNF-induced apoptosis reveals 550 me of functional conservation. Proc Natl Acad Sci USA 111(26): 9567-9572.
Creative Commons Attribution 4.0 International License

For possible submissions Click Here

\section{Submit Article}

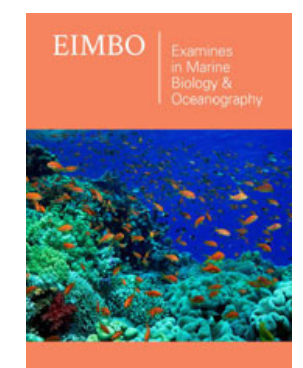

Examines in Marine Biology \& Oceanography

\section{Benefits of Publishing with us}

- High-level peer review and editorial services

- Freely accessible online immediately upon publication

- Authors retain the copyright to their work

- Licensing it under a Creative Commons license

- Visibility through different online platforms 